\title{
Effect of heat-killed Streptococcus thermophilus on type 2 diabetes rats
}

\author{
Xiangyang Gao ${ }^{\text {Equal first author, } 1}$, Fei Wang ${ }^{\text {Equal first author, } 1}$, Peng Zhao ${ }^{1,2}$, Rong Zhang ${ }^{1}$, Qiang Zeng ${ }^{\text {Corresp. } 1}$ \\ 1 Health Management Institute, The Second Medical Center of Chinese PLA General Hospital, Beijing, China \\ 2 Health Management Center, HangZhou Special Service Convalescent Center of Air Force, PLA, Hangzhou, China \\ Corresponding Author: Qiang Zeng \\ Email address: zengqianghospital@126.com
}

Background and Aims: The link between gut microbiota and type 2 diabetes (T2D) has been addressed by numerous studies. Streptococcus thermophilus from fermented milk products, has been used as a probiotic in previous research. However, whether heat-killed $S$. thermophilus can improve the glycaemic parameters of diabetic rats remains unanswered. In this study, we evaluated the effect of heat-killed $S$. thermophilus on T2D model rats and the potential mechanisms of the effect.

Methods: Zucker diabetic fatty (ZDF) rats were used to generate a diabetic rat model induced by feeding a high-fat diet. Heat-killed $S$. thermophilus were orally administered to normal and diabetic rats for 12 weeks. Intestinal microbiota analysis, histology analysis, oral glucose tolerance test (OGTT) and measurement of inflammatory factors were performed.

Results: We found that heat-killed $S$. thermophilus treatment reduced fasting blood glucose levels and alleviated glucose intolerance and total cholesterol in diabetic ZDF rats. Additionally, heat-killed $S$. thermophilus increased the IL-10 while reducing the levels of LPS, IL- 6 and TNF- $\alpha$ in diabetic ZDF rats. The heat-killed $S$. thermophilus treatment can normalize the structure of the intestinal and colon mucosal layer of diabetic rats. The characteristics of the gut microbiota in heat-killed $S$. thermophilus-treated and control rats were similar. At the genus level, the abundances of beneficial bacteria, including Ruminococcaceae, Veillonella, Coprococcus and Bamesiella, were all significantly elevated by heat-killed S. thermophilus treatment in ZDF diabetic rats.

Conclusion: Our study supports the hypothesis that treatment with heat-killed $S$. thermophilus could effectively improve glycaemic parameters in T2D model rats. In addition, the potential mechanisms underlying the protection maybe include changing the composition of gut microbiota, reinforcing the intestinal epithelial barrier and the immunity of the intestinal mucosa, decreasing the level of inflammation, and then reducing the insulin resistance. 
1 Effect of heat-killed Streptococcus thermophilus on type 2

2

3

4

5

6

7

8

\section{9}

\section{diabetes rats}

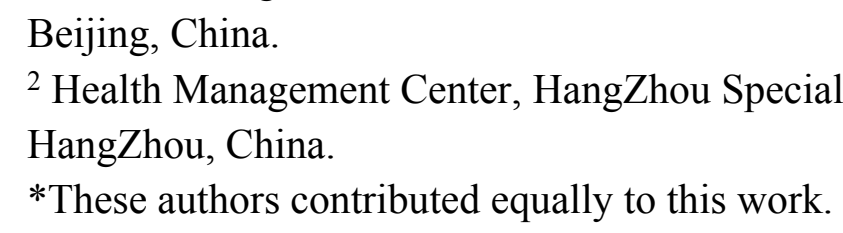




\section{Abstract}

41 Background and Aims: The link between gut microbiota and type 2 diabetes (T2D) has been addressed by numerous studies. Streptococcus thermophilus from fermented milk products, has been used as a probiotic in previous research. However, whether heat-killed $S$. thermophilus can

44

45

46 improve the glycaemic parameters of diabetic rats remains unanswered. In this study, we evaluated the effect of heat-killed S. thermophilus on T2D model rats and the potential mechanisms of the effect.

Methods: Zucker diabetic fatty (ZDF) rats were used to generate a diabetic rat model induced by feeding a high-fat diet. Heat-killed $S$. thermophilus were orally administered to normal and diabetic rats for 12 weeks. Intestinal microbiota analysis, histology analysis, oral glucose tolerance test (OGTT) and measurement of inflammatory factors were performed.

Results: We found that heat-killed $S$. thermophilus treatment reduced fasting blood glucose levels and alleviated glucose intolerance and total cholesterol in diabetic ZDF rats. Additionally, heat-killed $S$. thermophilus increased the IL-10 while reducing the levels of LPS, IL-6 and TNF$\alpha$ in diabetic ZDF rats. The heat-killed $S$. thermophilus treatment can normalize the structure of the intestinal and colon mucosal layer of diabetic rats. The characteristics of the gut microbiota in heat-killed $S$. thermophilus-treated and control rats were similar. At the genus level, the abundances of beneficial bacteria, including Ruminococcaceae, Veillonella, Coprococcus and Bamesiella, were all significantly elevated by heat-killed $S$. thermophilus treatment in ZDF diabetic rats.

Conclusion: Our study supports the hypothesis that treatment with heat-killed $S$. thermophilus could effectively improve glycaemic parameters in T2D model rats. In addition, the potential mechanisms underlying the protection maybe include changing the composition of gut microbiota, reinforcing the intestinal epithelial barrier and the immunity of the intestinal mucosa, decreasing the level of inflammation, and then reducing the insulin resistance.

\section{INTRODUCTION}

Diabetes is a chronic metabolic disease and an important cause of mortality and morbidity worldwide, the prevalence of which is dramatically increasing. The number of adults with diabetes, mostly Type 2 diabetes (T2D), has increased to 422 million around the world ${ }^{1}$. Diabetes and its complications account for more than 2 million deaths every year ${ }^{2}$.

Recently, immense evidence has been obtained linking T2D and gut microbiota. The significant correlations with specific gut microbes, bacterial genes, and metabolic pathways in T2D patients were showed by a human metagenome-wide association study ${ }^{3}$.The data from animal and human models also suggest that T2D is associated with a moderate degree ${ }^{4}$ to profound gut microbial dysbiosis ${ }^{5}$. Increasing evidence indicates that gut microbiota are strongly associated with diabetes development ${ }^{6-7}$. Other studies even show that gut microbiota markedly contribute to the incidence of $\mathrm{T}_{2} \mathrm{D}^{8-9}$. The dysbiosis of gut microbiota may damage the intestinal epithelial barrier, and increase the intestinal permeability, and thus promotes metabolic endotoxemia, and systemic inflammation ${ }^{10-11}$, leading to the development of insulin resistance ${ }^{12-}$ 
$80{ }^{13}$, thereby increasing the risk of developing T2 $\mathrm{D}^{14-15}$. These studies suggest that the gut 81 microbiota are potential targets for the treatment of T2D.

82 Probiotics have been proven to be effective in T2D. Administration of probiotics in a rat 83 model effectively inhibited gluconeogenesis in T2D ${ }^{16}$. Treatments with probiotics have been 84 demonstrated to be efficacious against tissue inflammation, and insulin resistance by modulating 85 the gut microbial structure ${ }^{17-18}$. However the efficacy in T2D subjects varies, depending on the 86 types and strains of probiotics.

87 Probiotics, as defined by the World Health Organization (WHO), are live microorganisms, 88

89

90

91

92

93

94

95

96

97

98

99

100

101

102

103

104

105

106

107

108

109

110

111

112

113

\section{Control rats}

115 Seven-week-old male Sprague-Dawley (SD) rats also were obtained from Charles River 116

117

118

119 many cases, probiotic preparations comprised of dead cells and their metabolites can also exert a biological response similar to that seen with live cells ${ }^{20-22}$. For example, both live and heat-killed Lactobacillus GG had a similar anti-inflammatory effect ${ }^{23}$.

Streptococcus thermophilus is classified as a lactic acid bacterium (LAB), and it is found in fermented milk products, and generally used in the dairy industry ${ }^{24}$. S. thermophilus scavenges reactive oxygen radicals ${ }^{25-26}$, thus demonstrating its antioxidant properties. S. thermophilus also shows immunomodulatory effects by stimulating the gut immune system ${ }^{27-28}$. And $S$. thermophilus has been used as a probiotic to help prevent developing insulin resistance in previous research ${ }^{29}$. However, to our knowledge, the question as to whether heat-killed $S$. thermophilus can improve glycaemic parameters remains unanswered. In addition, the potential mechanisms underlying the possible protection are still poorly understood. Therefore, the purpose of this research was to identify the beneficial effects of heat-killed S. thermophilus on diabetic rats and the potential mechanisms.

\section{MATERIALS AND METHODS}

\section{T2D animal model}

The Zucker diabetic fatty (ZDF) rats were used as a T2D model. ZDF rats have been an important model for studying the mechanism of treatment on T2D ${ }^{30-31}$. Seven-week-old male ZDF rats were purchased from Charles River (Beijing, China). After 1 week of acclimation, diabetes was then induced by feeding a high-fat diet of Purina5008 (17\% kcal fat and 26.5\% kcal protein, IPS Supplies, London, UK) for one month. Then, 12-week-old male ZDF rats were obtained, and fasting blood glucose $(\mathrm{FBG})>11.1 \mathrm{mmol} / \mathrm{L}$ was determined to be the standard concentration for the T2D model. (Beijing, China). After acclimating for 1 week, they were used as control rats.

Both the ZDF and SD rats were maintained at $22 \pm 2{ }^{\circ} \mathrm{C}$ with lights in an air-conditioned room with a 12-hour light/dark cycle, and were given free access to food and water. A standardized diet (kcal\%: 10\% fat, 20\% protein, and 70\% carbohydrate) was administered. All of the that confer a health benefit to the host, when administered in adequate amounts ${ }^{19}$. However, in 
120 experimental protocols were approved by the Animal Care Committee of the General PLA

121

122

123

124

125

126

127

128

129

130

131

132

133

134

135

136

137

138

139

140

141

142

143

144

145

146

147

148

149

150

151

152

153

154

155

156

157

158

159

Hospital Animal Ethics Committee (Project CPLAGHAE-20171228-01).

\section{Study design}

The diabetic ZDF rats were randomly divided into two groups: a heat-killed $S$. thermophilustreated diabetic group (DM + ST, KAWAI, Kawai Lactic Acid Bacteria Research Institute Co., Ltd., Japan, orally administered $0.21 \mathrm{~g}$ Kawai powder/kg body weight/day, $\mathrm{n}=5$ ) and an untreated diabetic group (DM, orally administered the same volume of normal saline, $\mathrm{n}=5$ ). Kawai powder contains $28.75 \%$ heat-killed $S$. thermophilus and $20.60 \%$ resistant dextrin, $20.00 \%$ isomaltooligosaccharide, $17.00 \%$ microcrystalline cellulose, $10.00 \%$ xylo-oligosaccharides, $2.55 \%$ Saccharomyces cerevisiae, and $1.10 \%$ lemon juice powder.

Control rats were randomly divided into an untreated control group (CON, administered normal saline, $\mathrm{n}=5)$ and a heat-killed S.thermophilus-treated control group (CON $+\mathrm{ST}$, orally administered 0.21 g Kawai powder $/ \mathrm{kg}$ body weight/day, $\mathrm{n}=5$ ). After treatment for 12 weeks, fresh stool samples were obtained by stimulating the anus, and they were frozen and stored at $80^{\circ} \mathrm{C}$ for subsequent analysis. After food deprivation for $12 \mathrm{~h}$, the rats were anesthetized, blood samples were collected from the aorta abdominalis, and then the rats were sacrificed.

\section{Tissue collection and histology analysis}

After rats were killed, the tissues of the ileum and colon were immediately excised, and then were cleaned with ice-cold phosphate-buffered saline solution. The tissues were fixed in $4 \%$ formalin solution, then embedded in paraffin before being cut into 4- $\mu \mathrm{m}$ slices, followed by hematoxylin-eosin (H\&E) staining for measurement of villi length and crypt depth (10 villi and 10 crypts per section) under a light microscope (SZX16, Olympus, Japan).

\section{Western blot analysis}

The ileum and colon tissues were homogenized in RIPA lysis buffer containing protease inhibitor cocktail (Roche,Indianapolis, IN, USA). Protein homogenates were separated on SDSPAGE gels and transferred to polyvinylidene difluoride membranes. After blocking for $1 \mathrm{~h}$ with $5 \%$ bovine serum albumin (BSA) in Tris-buffered saline with $0.1 \%$ Tween (TBST: $50 \mathrm{mM}$ Tris$\mathrm{HCl}, 150 \mathrm{mM} \mathrm{NaCl}, 0.1 \%$ Tween $20, \mathrm{pH} 7.4$ ), the membranes were incubated overnight with specific primary antibodies against Occludin(Abcam, Cambridge,UK), ZO-1 (Zonula occludens) (Santa Cruz Biotechnology, CA, USA), and $\beta$-actin (Zsbio, Beijing, China) at $4^{\circ} \mathrm{C}$. Then, the membranes were incubated for $1 \mathrm{~h}$ with the appropriate horseradish peroxidase (HRP)conjugated secondary antibodies (anti-rabbit or anti-mouse IgG-HRP) (Jackson ImmunoResearch Inc, West Grove, PA, USA), and the bands were detected by using enhanced chemiluminescence. The blots were scanned by a Bio-Rad ChemiDoc XRS and the intensity of each protein was quantified by Gel Image system V4.00 software (Tanon, Shanghai, China).

\section{Oral glucose tolerance test (OGTT)}

PeerJ reviewing PDF | (2019:01:34665:2:2:NEW 3 May 2019) 
160

161

162

163

164

165

166

167

168

169

170

171

172

173

174

175

176

177

178

179

180

181

182

183

184

185

186

187

188

189

190

191

192

193

194

195

196

197

198

199

At the end of the trial, an OGTT was performed after fasting for $12 \mathrm{~h}$. Glucose $(2 \mathrm{~g} / \mathrm{kg}$ body weight) was orally administered to the rats. The blood glucose levels which were obtained from the tail were recorded with a OneTouch UltraEasy glucometer (Johnson \& Johnson, New Brunswick, NJ, USA) before and 15, 30, 60, 90, and $120 \mathrm{~min}$ after the glucose load. The area under the curve (AUC) was calculated by using the linear trapezoid method ${ }^{32}$.

\section{Measurement of inflammatory factors, serum insulin, lipid profile, HOMA-IR, and HbA1c}

After food deprivation for $12 \mathrm{~h}$, rat serum was obtained to analyze inflammatory factors (interleukin 6 (IL-6), interleukin 10 (IL-10), tumor necrosis factor (TNF)- $\alpha$, and lipopolysaccharide (LPS) ( ELISA, Elabscience, Wuhan, China), insulin (ELISA, Millipore,Billerica, MA, USA), total cholesterol (TC), triglyceride (TG, oxidase method; InTec Products, Fujian, China), high-density lipoprotein cholesterol concentrations (HDL-C), and lowdensity lipoprotein cholesterol concentrations (LDL-C, direct method, InTec Products, Fujian, China), according to the manufacturer's instruction. The homeostasis model assessment of insulin resistance (HOMA-IR) was calculated by using the following formula: fasting blood glucose $(\mathrm{FBG}, \mathrm{mmol} / \mathrm{L}) \times$ fasting serum insulin $(\mu \mathrm{IU} / \mathrm{ml}) / 22.5$. Rat plasma was also analyzed for HbAlc (Immunoturbidimetry, InTec Products, Fujian, China).

\section{Intestinal microbiota analysis}

DNA extractions from total fecal bacteria were obtained using a QIAamp Stool DNA Extraction Kit (Qiagen, Valencia, CA, USA) according to the manufacturer's instructions. The microbial 16S rRNA hypervariable regions V3-V4 were amplified with indexes and adaptor-linked universal primers (341F: 50-ACTCCTACGGGAGGCAGCAG-30, 806R: 50GGACTACHVGGGTWTCTAA-30T). PCR was performed by using a KAPA HiFi Hotstart PCR kit (KAPA Biosystems, Wilmington, DE, USA) with high fidelity enzyme in triplicate. Amplicon libraries were quantified using a Qubit 2.0 Fluorometer(Thermo Fisher Scientific, Waltham, MA, USA) and then sequenced on the Illumina HiSeq 2500 platform (Illumina, San Diego, CA, USA) for 250-bp paired-end reads. After discarding the singletons and removing chimeras, operational taxonomic units (OTUs) were generated using USEARCH (v7.0.1090) at $97 \%$ similarity by clustering the tags. Final OTUs were taxonomically classified based on the RDP classifier version 2.2 algorithm using the GreenGene database. Alpha diversity (Chao1, Shannon, Simpson) and beta diversity (principal coordinates analysis (PCoA) plots) were analyzed using QIIME version 1.7.0. In addition, a $t$ test was performed to compare the differences between groups by using STAMP. The relative abundance of bacteria is expressed as the percentage $(\%)$.

\section{Data analysis}

The data are expressed as the mean \pm standard deviation (SD). When the data were normal and variances were equal, differences among the groups were analyzed using $t$ test. For non-normal distribution data, In transformation was carried out before analysis. A $\mathrm{p}$ value $<0.05$ was 
200

201

202

203

204

205

206

207

208

209

210

211

212

213

214

215

216

217

218

219

220

221

222

223

224

225

226

227

228

229

230

231

232

233

234

235

236

237

238

239

considered statistically significant. All of the statistical analyses were performed using the Statistical Package for Social Sciences version 17 software (SPSS Inc., Chicago, IL, USA).

\section{Results}

\section{Body weight}

The body weights of the heat-killed $S$. thermophilus-treated diabetic rats were comparable with those of the untreated diabetic rats $(\mathrm{P}>0.05$, Table 1$)$. There was also no significant difference in body weight between the CON group and $\mathrm{CON}+\mathrm{ST}$ group $(\mathrm{P}>0.05$, Table 2$)$.

\section{Fasting blood glucose level and glucose tolerance}

The heat-killed $S$. thermophilus treatment reduced fasting blood glucose (FBG) levels in diabetic rats $(\mathrm{P}<0.05$, Figure 1$)$. The blood glucose levels significantly decreased before and 15,60 , and 90 min after glucose load ( $\mathrm{P}<0.05$, Figure 1$)$ in the $\mathrm{DM}+\mathrm{ST}$ group as compared to those in the DM group according to the OGTT. At the time points of 30 and $120 \mathrm{~min}$ after the glucose load, the blood glucose levels were lower in the DM+ST group than those in DM group, but the differences were not significant $(\mathrm{P}>0.05$, Figure 1). Compared with the DM group, the glucose area under the curve (AUC) for the OGTT in the DM+ST group exhibited a reduced glucose AUC by $14.7 \%(\mathrm{P}<0.05$, Figure 2$)$.

According to the OGTT, although there were no significant differences before and 15, and 120 min after the glucose load between the CON group and CON+ST group, the blood glucose levels significantly decreased 30 and 60 min after the glucose load $(\mathrm{P}<0.05$, Figure 3$)$ in the $\mathrm{CON}+\mathrm{ST}$ group as compared to those in the $\mathrm{CON}$ group. The $\mathrm{CON}+\mathrm{ST}$ group exhibited a reduced glucose AUC by $18.2 \%(\mathrm{P}<0.05$, Figure 4$)$ for the OGTT, compared with the CON group.

\section{Fasting insulin, HbA1c, and HOMA-IR}

The heat-killed $S$. thermophilus treatment reduced serum insulin levels, HbA1c, and HOMA-IR ( $\mathrm{P}<0.05$, Table 1) in ZDF diabetic rats. However, compared to the CON group, the heat-killed $S$. thermophilus treatment rats failed to produce significantly lower serum insulin levels or HOMA-IR in the CON+ST group ( $<<0.05$, Table 2$)$.

\section{Serum biochemical parameters}

There were no significant differences in the serum creatinine, alanine aminotransferase (ALT), carbamide, or uric acid levels between the two groups in ZDF diabetic rats. The level of TC significantly increased in the DM+ST group, while the heat-killed $S$. thermophilus treatment did not significantly reduce the TG, HDL-C, or LDL-C levels in diabetic rats $(\mathrm{P}<0.05$, Table 1$)$. In contrast, there were no significant difference in serum biochemical parameters in the $\mathrm{CON}+\mathrm{ST}$ group compared to the CON group $(\mathrm{P}>0.05$, Table 1$)$.

\section{Inflammatory factors}

Peer] reviewing PDF | (2019:01:34665:2:2:NEW 3 May 2019) 
240 Compared with the DM group, the inflammatory factors LPS, IL-6, and TNF- $\alpha$ significantly 241 decreased and IL-10 significantly increased in the DM+ST group $(\mathrm{P}<0.05$, Table 1$)$. There were 242 no significant differences in the inflammatory factors between the CON+ST group and CON 243 group $(\mathrm{P}>0.05$, Table 2$)$.

244

245

246

247

248

249

250

251

252

253

254

255

256

257

258

259

260

261

262

263

264

265

266

267

268

269

270

271

\section{Histological analysis}

We examined the heat-killed S. thermophilus effects on the villi length and crypt depth in the ileum. In the diabetic rats, the intestinal mucosal layer was characterized by disturbed mucosal architecture, shortened villi, blunted villus tips, and inflammatory cell infiltration. In the DM+ST group, oral administration of $S$. thermophilus restored the normal structure of the intestinal mucosal layer (Figure 5). The length of villi and depth of crypts in the DM+ST group were significantly increased compared to those in DM group (Figure 6). Additionally, goblet cells were counted per villus/crypt in the ileum. The ileum exhibited a significant increase in total goblet cell number after treatment with $S$. thermophilus $(39.2 \pm 4.2$ vs $20.9 \pm 5.0, \mathrm{p}<0.05)$. Similar findings were also seen in the colonic tissues (Figure 5 and 7). There were no differences in the villi length and crypt depth and the numbers of goblet cells between the CON and CON+ST groups (Figure 8).

\section{Western blot analysis}

To explore the mechanisms underlying the heat-killed $S$. thermophilus effects on the barrier function, the expression levels of Occludin and ZO-1 proteins were determined by Western blot analysis. The results showed that Occludin and ZO-1 proteins in the DM+ST group were significantly elevated compared with the DM group both in the ileum and colon tissues (1.76fold increases for Occludin and 2.29-fold increases for ZO-1 in the ileum tissues; 1.64-fold increases for Occludin and 1.46-fold increases for ZO-1 in the colon tissues, vs. the DM group) (Figure 9). There were no differences in the expression levels of Occludin and ZO-1 in the ileum or colon tissues between the $\mathrm{CON}$ and $\mathrm{CON}+\mathrm{ST}$ groups (Figure 10).

\section{Characterization of gut microbiota}

In ZDF diabetic rats, the richness of the gut microbiota was increased in the DM+ST group compared with the DM group; however, the difference was not significant, as shown in Table 3.

272 in Fig.11, to assess the bacterial community between two groups, a principal coordinate analysis

273 (PCoA) for the unweighted UniFrac distance matrices was performed. The first two principal

274

275 coordinates of PCoA (components 1 and 2) were separated into DM+ST and DM groups, which shared overlapping regions. As in the above analysis, the DM+ST and DM groups, and

276

277 $\mathrm{COM}+\mathrm{ST}$ and CON groups exhibited similar alpha and beta diversities in the gut microbiota.

278 The results indicate that the treatment with heat-killed S. thermophilus could not improve the richness of the gut microbiota. 
279

280

281

282

283

\section{4}

285

286

287

288

289

290

291

292

293

294

295

296

297

298

299

300

301

302

303

304

305

306

307

308

309

310

311

312

313

314

315

316

317

318

At the genus level, the abundance of Ruminococcaceae, Veillonella, Coprococcus, and Bamesiella was significantly elevated by heat-killed S. thermophilus treatment in ZDF diabetic rats $(\mathrm{P}<0.05$, Fig. 12), whereas Phascolarctobacterium and Dorea abundances were reduced by heat-killed $S$. thermophilus treatment in SD control rats $(\mathrm{P}<0.05$, Fig. 13).

\section{DISCUSSION}

In this study, heat-killed $S$. thermophilus bacteria were administered to ZDF T2D rats to test whether they have a protective effect. The ZDF diabetic rat is a well-characterized model of T2D, and the rats has been used in many studies to examine human T2D pathophysiology and the effects of therapeutic options ${ }^{33-34}$. Interestingly, we found that the heat-killed $S$. thermophilus treatment effectively moderated insulin resistance and glucose intolerance in the ZDF T2D rat model. To our knowledge, this is the first report about the effect of the heat-killed $S$. thermophilus treatment on glycaemic parameters of diabetic rats. Many previous studies focused on the relation between live S. thermophilus and human. For instance, a multispecies probiotic supplement consisting of $S$. thermophilus reduced the fasting plasma glucose and serum highsensitivity C-reactive protein, and increased plasma total glutathione ${ }^{35}$. And the probiotic mix VSL\#3, which contains $S$. thermophilus, increased insulin sensitivity, and affected the composition of gut microbiota ${ }^{36}$. So our work provides new insights into the function of the heatkilled S. thermophilus.

Another effect of the heat-killed $S$. thermophilus treatment is that of significantly reducing the level of TC in the diabetic rats used in this study. The effect of removing cholesterol probably occurs by two mechanisms: binding cholesterol to the cell surface ${ }^{37-38}$ or deconjugating bile salts to prevent their recycling ${ }^{39-40}$.

In addition, we found that the heat-killed $S$. thermophilus treatment increased the abundance of Ruminococcaceae, Veillonella, Coprococcus and Barnesiella at the genus level in diabetic rats. The normal gut microbiota has many functions, such as protection against pathogens, immunomodulation, maintenance of the gut mucosal barrier structural integrity, and nutrient and drug metabolism ${ }^{41}$. As a member of short chain fatty acid producers, Ruminococcaceae is inversely correlated with increased intestinal permeability ${ }^{42}$, and alcoholic cirrhosis ${ }^{43}$. The abundance of Ruminococcaceae has been observed to significantly increase after treatment with fucoidan ${ }^{44}$. Coprococcus is a butyrate-producing genera ${ }^{45}$. Dietary intervention including extensively hydrolyzed casein formula supplemented with Lactobacillus rhamnosus $G G$ to enrich Coprococcus could accelerate tolerance acquisition in infants who are allergic to milk ${ }^{46}$. Veillonella are normal bacteria found in the intestines of mammals, that are well known for their lactate fermenting abilities. A positive association has been found between lactose levels and the abundance of the Veillonella genus ${ }^{47}$. Anaerobic bacteria belonging to the Barnesiella genus enable clearance of intestinal colonization by the highly antibiotic-resistant bacterium vancomycin-resistant Enterococcus ${ }^{48}$. When compared with high-fat, high-sucrose-fed mice, Barnesiella spp are the main discriminative feature of chow-fed mice ${ }^{49}$. Therefore, Barnesiella may have a beneficial impact on host metabolism. 
Many effects of probiotics are mediated through immune regulation and through the balance of anti-inflammatory and pro-inflammatory cytokines. In this study, the heat-killed $S$. thermophilus treatment significantly decreased the inflammatory factors LPS, IL-6, and TNF- $\alpha$, and increased IL-10. From the membranes of gram-negative bacteria, LPS penetrates into the blood via impaired permeability of the intestinal mucosa, which is caused by the reduced expression of adhesion and tight junction proteins ${ }^{50}$. Then, LPS triggers a strong proinflammatory reaction and secretion of proinflammatory cytokines from the host cells, followed by metabolic endotoxemia ${ }^{51}$. Metabolic endotoxemia increases systemic inflammation and impairs insulin sensitivity in both adipose tissue and the liver ${ }^{52}$. It can also impair insulin signaling by inducing endoplasmic reticulum stress and the activity of a histone acetyltransferase ${ }^{53}$. The high circulating LPS characterizes both incident and prevalent diabetes in a clinical observation also suggests the relevance of this putative mechanism to humans ${ }^{54}$. As the product of pro-inflammatory cells, IL-6 is involved in many biological processes, such as the host response to acute-phase reactions, haematopoiesis, enteric pathogens, and terminal differentiation of B-lymphocytes ${ }^{55}$. IL-10 is a potent deactivator of macrophage/monocyte proinflammatory cytokine synthesis ${ }^{56}$, such as downregulation of TNF- $\alpha$ secretion by macrophages ${ }^{57}$.

It was also found that the heat-killed $S$. thermophilus treatment protected the intestinal barrier. In our study, an increased ileum villus/crypt length and number of goblet cells were observed in the DM+ST group with $S$. thermophilus administration compared with the DM group. This is consistent with previous studies reporting that probiotic administration markedly deepened jejunal crypts in healthy rats $^{58}$, and both villus and crypt were lengthened after treatment by emu oil ${ }^{59}$. The main role of goblet cells is to protect the mucous membrane by secreting mucus ${ }^{60}$. There is a strong association between intestinal flora and secretion of mucin ${ }^{61}$, as goblet cells may be regulated by interactions between the gastrointestinal mucosa and specific bacterial peptides ${ }^{62}$. The results of our study also showed that the Occludin and ZO-1 proteins in the DM+ST group were significantly elevated compared with the DM group both in the ileum and colon tissues. Intestinal barrier integrity is maintained by the tight junctions those are made of transmembrane, scaffold and adaptor proteins. Occludin is transmembrane protein embedded in the intracellular actin through attachment to adaptor protein ZO- ${ }^{63}$. It is widely reported that commensal bacteria have profound effects on epithelial integrity and permeability, particularly, on tight junctions maintenance ${ }^{64}$. A dysbiosis adversely enhances intestinal permeability by modulating the expression of epithelial 1 tight junction proteins ZO-1 and Occludin ${ }^{65}$. The mucosal barrier is very important for protecting the host tissue from damage that is mediated by toxic products or luminal pathogens obtained from food or pathogenic bacteria, while allowing uptake of nutrients at the same time. A previous study showed that feeding fermented milk produced by $S$. thermophilus and Bifidobacterium breve could reinforce the intestinal barrier ${ }^{66}$. Another study also showed that live S. thermophilus significantly increased the transepithelial electrical resistance in the intestinal Caco-2 cell monolayer by enhancement 
358

359

360

361

362

363

364

365

366

367

368

369

370

371

372

373

374

375

376

377

378

379

380

381

382

383

384

385

386

387

388

389

390

391

392

393

394

395

396

(actinin, occludin) or maintenance(actin, ZO-1) of cytoskeletal and tight junctional protein phosphorylation ${ }^{67}$.

In the current study, the S. thermophilus used was heat-killed instead of live cells. Both live and dead cells are capable of generating a biological response ${ }^{20}$. Our result is consistent with a recent study which shows that pasteurized Akkermansia muciniphila is able to ameliorate highfat diet induced dysglycemia ${ }^{68}$. In a meta-analysis, modified (heat-killed or sonicated) probiotics were found to have effects similar to those of the living probiotics in most trials ${ }^{69}$. The effects of heat-killed probiotics may be attributed to the dead cells and/or their metabolites. For example, metabolites released by $S$. thermophilus exerted an anti-TNF- $\alpha$ effect and were capable of crossing the intestinal barrier ${ }^{70}$. Besides, a recombinant protein isolated from the A. muciniphila membrane can lead to an improved gut barrier ${ }^{68}$. Notably, even A. muciniphila-derived extracellular vesicles can decrease gut permeability by regulating the tight junctions ${ }^{71}$. It has also been documented that bacterial muramyl dipeptide reduces inflammation and promotes insulin signaling in the state of metabolic endotoxemia, and glycemia ${ }^{72}$. As a bacterial metabolite, indole is able to counteract the pro-inflammatory and metabolism-altering effects of LPS in the liver ${ }^{73}$. Similarly, SCFAs can improve barrier function ${ }^{74}$, decrease inflammation, and promote the metabolism of lipids and glucose ${ }^{75-76}$. In addition, microbiota-derived succinate can also improve glucose metabolism by acting on intestinal gluconeogenesis ${ }^{77}$.

One limitation to widespread use of probiotic therapy is the concern regarding adverse effects, which may cause some pathology of their own ${ }^{78-79}$. Compared with live probiotics, heatkilled probiotics are safer for purposes such as application in immunosuppressed patients and children ${ }^{80}$. Another problem with live probiotics is that they would have to survive proteolytic enzymes and the low $\mathrm{pH}$ of stomach acid. The recovery rate of total $S$. thermophilus from the terminal ileum of minipigs was very low after digesting a certain amount of live cells ${ }^{81}$. The preparation and administration of heat-killed probiotics are convenient compared to live probiotics $^{82}$. Products based on dead cells are easier to standardize, and store, and they also have a long shelf-life ${ }^{55}$. Therefore, heat-killed probiotics may be a promising and safer alternative to live probiotics.

After the analysis of numerous studies, it was proposed that there may be a bacteria mucosal immunity-inflammation-diabetes (BMID) axis, through which herbal monomers and formulae improve diabetes ${ }^{83}$. In this study, heat-killed S. thermophilus may also affect diabetes through the BMID axis by increasing the abundance of beneficial bacteria, protecting the intestinal epithelial barrier, and suppressing IL-6, LPS, and TNF- $\alpha$ secretion, and the end result is moderation of insulin tolerance.

\section{CONCLUSION}

Our study supports the hypothesis that treatment with heat-killed $S$. thermophilus could effectively improve the glycaemic parameters of T2D model rats. In addition, the potential mechanisms underlying the protection may consist of changing the composition of gut 
397

398

399

400

401

402

403

404

405

406

407

408

409

410

411

412

413

414

415

416

417

418

419

420

421

422

423

424

425

426

427

428

429

430

431

432

433

434

435

436

437

438

439

440

441

442

443

444

microbiota, reinforcing the intestinal epithelial barrier and the immunity of the intestinal mucosa, decreasing the level of inflammation, and then reducing insulin resistance.

\section{Acknowledgements}

We are very grateful to CapitalBio Technology Co., Ltd. for excellent technical assistance with 16 s sequencing experiments, and thank LetPub (www.letpub.com) for its linguistic assistance during the preparation of this manuscript.

\section{References}

1. Zhou, B.; Lu, Y.; Hajifathalian, K.; Bentham, J.; Di Cesare, M.; Danaei, G.; Bixby, H.; J Cowan, M.; Ali, M.; Taddei, C.; Lo, W.-C.; Reis-Santos, B.; A Stevens, G.; M Riley, L.; Miranda, J. J.; Bjerregaard, P.; Rivera, J.; Fouad, H.; Ma, G.; Ezzati, M., Worldwide trends in diabetes since 1980: a pooled analysis of 751 population-based studies with 4.4 million participants. 2016; Vol. 387, p 1513-1530.

2. Li, C.; Li, X.; Han, H.; Cui, H.; Peng, M.; Wang, G.; Wang, Z., Effect of probiotics on metabolic profiles in type 2 diabetes mellitus: A meta-analysis of randomized, controlled trials. Medicine 2016, 95 (26), e4088.

3. Nadja, L.; Vogensen, F. K.; Berg, F. W. J. V. D.; Dennis Sandris, N.; Anne Sofie, A.; Pedersen, B. K.; Waleed Abu, A. S.; S?Rensen, S. R. J.; Hansen, L. H.; Mogens, J., Gut microbiota in human adults with type 2 diabetes differs from non-diabetic adults. PloS one 2010, 5 (2), e9085.

4. Qin, J.; Li, Y.; Cai, Z.; Li, S.; Zhu, J.; Zhang, F.; Liang, S.; Zhang, W.; Guan, Y.; Shen, D.; Peng, Y.; Zhang, D.; Jie, Z.; Wu, W.; Qin, Y.; Xue, W.; Li, J.; Han, L.; Lu, D.; Wu, P.; Dai, Y.; Sun, X.; Li, Z.; Tang, A.; Zhong, S.; Li, X.; Chen, W.; Xu, R.; Wang, M.; Feng, Q.; Gong, M.; Yu, J.; Zhang, Y.; Zhang, M.; Hansen, T.; Sanchez, G.; Raes, J.; Falony, G.; Okuda, S.; Almeida, M.; LeChatelier, E.; Renault, P.; Pons, N.; Batto, J. M.; Zhang, Z.; Chen, H.; Yang, R.; Zheng, W.; Li, S.; Yang, H.; Wang, J.; Ehrlich, S. D.; Nielsen, R.; Pedersen, O.; Kristiansen, K.; Wang, J., A metagenome-wide association study of gut microbiota in type 2 diabetes. Nature 2012, 490 (7418), 55-60.

5. Tilg, H.; Moschen, A. R., Microbiota and diabetes: an evolving relationship. Gut 2014, 63 (9), 1513-1521.

6. Haro, C.; Montes-Borrego, M.; Rangel-Zúñiga, O. A.; Alcalá-Díaz, J. F.; GómezDelgado, F.; Pérez-Martínez, P.; Delgado-Lista, J.; Quintana-Navarro, G. M.; Tinahones, F. J.; Landa, B. B., Two healthy diets modulate gut microbial community improving insulin sensitivity in a human obese population. Journal of Clinical Endocrinology \& Metabolism 2015, 101 (1), jc20153351.

7. Mejía-León, M. E.; Barca, A. M. C. D. L., Diet, Microbiota and Immune System in Type 1 Diabetes Development and Evolution. Nutrients 2015, 7 (11), 9171-9184.

8. Baothman, O. A.; Zamzami, M. A.; Taher, I.; Abubaker, J.; Abu-Farha, M., The role of Gut Microbiota in the development of obesity and Diabetes. Lipids in Health \& Disease 2016, 15 (1), 1-8.

9. Ningwen, T.; F Susan, W.; Li, W., The role of gut microbiota in the development of type 1, type 2 diabetes mellitus and obesity. Reviews in Endocrine \& Metabolic Disorders 2015, 16 (1), 55-65.

10. Prattichizzo, F.; Giuliani, A.; Mensà, E.; Sabbatinelli, J.; De Nigris, V.; Rippo, M. R.; La Sala, L.; Procopio, A. D.; Olivieri, F.; Ceriello, A., Pleiotropic effects of metformin: Shaping the microbiome to manage type 2 diabetes and postpone ageing. Ageing research reviews 2018, $48,87-98$. 
445 11. Winer, D. A.; Luck, H.; Tsai, S.; Winer, S., The Intestinal Immune System in Obesity and 446 Insulin Resistance. Cell metabolism 2016, 23 (3), S1550413116000371.

447 12. Jorge, H. M.; Eran, E.; Chengcheng, J.; Liming, H.; Mehal, W. Z.; Till, S.; Thaiss, C. A.; 448 Kau, A. L.; Eisenbarth, S. C.; Jurczak, M. J., Inflammasome-mediated dysbiosis regulates 449 progression of NAFLD and obesity. Nature 2012, 482 (7384), 179-185.

450 13. Boulange, C.; Neves, A. L.; Chilloux, J.; Nicholson, J.; Dumas, M.-E., Impact of the gut 451 microbiota on inflammation, obesity, and metabolic disease. 2016; Vol. 8.

452 14. Pedersen, H. K.; Gudmundsdottir, V.; Nielsen, H. B.; Hyotylainen, T.; Nielsen, T.; 453 Jensen, B. A.; Forslund, K.; Hildebrand, F.; Prifti, E.; Falony, G.; Le Chatelier, E.; Levenez, F.; 454 Dore, J.; Mattila, I.; Plichta, D. R.; Poho, P.; Hellgren, L. I.; Arumugam, M.; Sunagawa, S.; 455 Vieira-Silva, S.; Jorgensen, T.; Holm, J. B.; Trost, K.; Meta, H. I. T. C.; Kristiansen, K.; Brix, S.; 456 Raes, J.; Wang, J.; Hansen, T.; Bork, P.; Brunak, S.; Oresic, M.; Ehrlich, S. D.; Pedersen, O., 457 Human gut microbes impact host serum metabolome and insulin sensitivity. In Nature, 2016; 458 Vol. 535, pp 376-81.

459 15. Hartstra, A. V.; Nieuwdorp, M.; Herrema, H., Interplay between gut microbiota, its 460 metabolites and human metabolism: Dissecting cause from consequence. 2016; Vol. 57.

461 16. $\quad$ Amandine, E.; Clara, B.; Lucie, G.; Ouwerkerk, J. P.; Céline, D.; Bindels, L. B.; Yves, G.; 462 Muriel, D.; Muccioli, G. G.; Delzenne, N. M., Cross-talk between Akkermansia muciniphila and 463 intestinal epithelium controls diet-induced obesity. Proceedings of the National Academy of 464 Sciences of the United States of America 2013, 110 (22), 9066-9071.

465 17. Angela, M. P.; Alexander, N.; Yolanda, S., Bifidobacterium pseudocatenulatum CECT 4667765 Reduces Obesity-Associated Inflammation by Restoring the Lymphocyte-Macrophage 467 Balance and Gut Microbiota Structure in High-Fat Diet-Fed Mice. PloS one 2015, 10 (7), 468 e0126976-.

469 18. Na-Ri, S.; June-Chul, L.; Hae-Youn, L.; Min-Soo, K.; Tae Woong, W.; Myung-Shik, L.; $470 \mathrm{Jin}-W o o$, B., An increase in the Akkermansia spp. population induced by metformin treatment 471 improves glucose homeostasis in diet-induced obese mice. Gut 2014, 63 (5), 727-735.

472 19. FAO/WHO, Report on joint FAO/WHO expert consultation on evaluation of health and 473 nutritional properties

474 of probiotics in food including powder milk with live lactic acid bacteria. Available from: URL: 475 http://www.fao.org./es/ESN/Probio/probio.htm 2001.

476 20. Dotan, I.; Rachmilewitz, D., Probiotics in inflammatory bowel disease: possible 477 mechanisms of action. Curr.opin.gastroenterol 2005, 21 (4), 426.

478 21. Sashihara, T.; Sueki, N.; Ikegami, S., An Analysis of the Effectiveness of Heat-Killed 479 Lactic Acid Bacteria in Alleviating Allergic Diseases. Journal of dairy science 2006, 89 (8), 28464802855.

$481 \quad 22 . \quad$ Liyan, Z.; Nan, L.; Ricardo, C.; Josef, N., Alive and dead Lactobacillus rhamnosus GG 482 decrease tumor necrosis factor-alpha-induced interleukin-8 production in Caco-2 cells. Journal 483 of Nutrition 2005, 135 (7), 1752-6.

484 23. Ehud, B.; Felix, M.; Marisa, H.; Abraham, W., Lactobacillus GG bacteria ameliorate 485 486 arthritis in Lewis rats. Journal of Nutrition 2004, 134 (8), 1964.

24. Kilic, A. O.; Pavlova Sima, W. G.; Tao, L., ANALYSIS OF LACTOBACILLUS PHAGES AND BACTERIOCINS IN AMERICAN DAIRY PRODUCTS AND CHARACTERIZATION OF A PHAGE ISOLATED FROM YOGURT. Applied \& Environmental Microbiology 1996, 62 (6), 2111-2116.

25. Lin, M. Y.; Yen, C. L., Antioxidative ability of lactic acid bacteria. J Agric Food Chem 1999, 47 (4), 1460-1466.

26. Bruno-Barcena, J. M.; Andrus, J. M.; Libby, S. L.; Klaenhammer, T. R.; Hassan, H. M., Expression of a Heterologous Manganese Superoxide Dismutase Gene in Intestinal Lactobacilli Provides Protection against Hydrogen Peroxide Toxicity. Applied \& Environmental Microbiology 2004, 70 (8), 4702. 
496

497

498

499

500

501

502

503

504

505

506

507

508

509

510

511

512

513

514

515

516

517

518

519

520

521

522

523

524

525

526

527

528

529

530

531

532

533

534

535

536

537

538

539

540

541

542

543

544

545

546

27. Donkor, O. N.; Ravikumar, M.; Proudfoot, O.; Day, S. L.; Apostolopoulos, V.; Paukovics, G.; Vasiljevic, T.; Nutt, S. L.; Gill, H., Cytokine profile and induction of T helper type 17 and regulatory $T$ cells by human peripheral mononuclear cells after microbial exposure. Clinical \& Experimental Immunology 2012, 167 (2), 282-295.

28. Delorme, C., Safety assessment of dairy microorganisms: Streptococcus thermophilus 々 3 . International journal of food microbiology 2008, 126 (3), 274-277.

29. Asemi, Z.; Samimi, M.; Tabassi, Z.; Rad, M. N.; Foroushani, A. R.; Khorammian, H.; Esmaillzadeh, A., Effect of daily consumption of probiotic yoghurt on insulin resistance in pregnant women: a randomized controlled trial. Pakistan Journal of Biological Sciences Pjbs 2013, 60 (1), 71-74.

30. Finegood, D.; McArthur, M.; Kojwang, D.; Thomas, M.; Topp, B.; Leonard, T.; Buckingham, R., Beta-cell mass dynamics in Zucker diabetic fatty rats. Rosiglitazone prevents the rise in net cell death. Diabetes 2001, 50 (5), 1021-1029.

31. Leonard, B. L.; Watson, R. N.; Loomes, K. M.; Phillips, A. R. J.; Cooper, G. J., Insulin resistance in the Zucker diabetic fatty rat: a metabolic characterisation of obese and lean phenotypes. Acta Diabetologica 2005, 42 (4), 162-170.

32. Zhang, Q.; Sun, X.; Xiao, X.; Zheng, J.; Li, M.; Yu, M.; Ping, F.; Wang, Z.; Qi, C.; Wang, T., Maternal Chromium Restriction Leads to Glucose Metabolism Imbalance in Mice Offspring through Insulin Signaling and Wnt Signaling Pathways. International journal of molecular sciences 2016, 17 (10), 1767-.

33. Ferreira, L.; Teixeira-De-Lemos, E.; Pinto, F.; Parada, B.; Mega, C.; Vala, H.; Pinto, R.; Garrido, P.; Sereno, J.; Fernandes, R., Effects of sitagliptin treatment on dysmetabolism, inflammation, and oxidative stress in an animal model of type 2 diabetes (ZDF rat). Mediators of Inflammation, 2010,(2010-05-31) 2010, 2010 (1), 60-68.

34. Christos, T.; Wookey, P. J.; Riccardo, C.; Sof, A.; Thomas, M. C.; Cooper, M. E., Improved islet morphology after blockade of the renin- angiotensin system in the ZDF rat. Diabetes 2004, 53 (4), 989-997.

35. Asemi, Z.; Zare, Z.; Shakeri, H.; Sabihi, S. S.; Esmaillzadeh, A., Effect of multispecies probiotic supplements on metabolic profiles, hs-CRP, and oxidative stress in patients with type 2 diabetes. Annals of nutrition \& metabolism 2013, 63 (1-2), 1-9.

36. Rajkumar, H.; Mahmood, N.; Kumar, M.; Varikuti, S. R.; Challa, H. R.; Myakala, S. P., Effect of probiotic (VSL\#3) and omega-3 on lipid profile, insulin sensitivity, inflammatory markers, and gut colonization in overweight adults: a randomized, controlled trial. Mediators of inflammation 2014, 2014 (9), 348959.

37. Kimoto, H.; Ohmomo, S.; Okamoto, T., Cholesterol Removal from Media by Lactococci. Journal of dairy science 2002, 85 (12), 3182-3188.

38. Liong, M. T.; Shah, N. P., Acid and bile tolerance and cholesterol removal ability of lactobacilli strains. Journal of dairy science 2005, 88 (1), 55-66.

39. Iyer, R.; Tomar, S. K.; Maheswari, T. U.; Singh, R., Streptococcus thermophilus strains: Multifunctional lactic acid bacteria. International Dairy Journal 2010, 20 (3), 133-141.

40. Kim, S.-J.; Hoon Park, S.; Sin, H.-S.; Jang, S.-H.; Lee, S.-W.; Kim, S.-Y.; Kwon, B.; Yu, K.-Y.; Kim, S. Y.; Kwon Yang, D., Hypocholesterolemic Effects of Probiotic Mixture on DietInduced Hypercholesterolemic Rats. 2017; Vol. 9, p 293.

41. Jandhyala, S. M.; Talukdar, R.; Subramanyam, C.; Vuyyuru, H.; Sasikala, M.; Reddy, D. $\mathrm{N}$., role of the normal gut microbiota. World journal of gastroenterology 2015, 21 (29), 87878803.

42. Leclercq, S.; Matamoros, S.; Cani, P. D.; Neyrinck, A. M.; Jamar, F.; Starkel, P.; Windey, K.; Tremaroli, V.; Backhed, F.; Verbeke, K.; de Timary, P.; Delzenne, N. M., Intestinal permeability, gut-bacterial dysbiosis, and behavioral markers of alcohol-dependence severity. Proceedings of the National Academy of Sciences of the United States of America 2014, 111 (42), E4485-93.

Peer] reviewing PDF | (2019:01:34665:2:2:NEW 3 May 2019) 
547 43. Bajaj, J. S.; Heuman, D. M.; Hylemon, P. B.; Sanyal, A. J.; White, M. B.; Monteith, P.;

548 Noble, N. A.; Unser, A. B.; Daita, K.; Fisher, A. R.; Sikaroodi, M.; Gillevet, P. M., Altered profile

549

550

551

552

553

554

555

556

557

558

559 of human gut microbiome is associated with cirrhosis and its complications. Journal of hepatology 2014, 60 (5), 940-7.

44. Shang, Q.; Shan, X.; Cai, C.; Hao, J.; Li, G.; Yu, G., Dietary fucoidan modulates the gut microbiota in mice by increasing the abundance of Lactobacillus and Ruminococcaceae. Food \& function 2016, 7 (7), 3224.

45. Fujio-Vejar, S.; Vasquez, Y.; Morales, P.; Magne, F.; Vera-Wolf, P.; Ugalde, J. A.; Navarrete, P.; Gotteland, M., The Gut Microbiota of Healthy Chilean Subjects Reveals a High Abundance of the Phylum Verrucomicrobia. Frontiers in microbiology 2017, 8, 1221.

46. Berni Canani, R.; Sangwan, N.; T Stefka, A.; Nocerino, R.; Paparo, L.; Aitoro, R.; Calignano, A.; A Khan, A.; Jack, G.; R Nagler, C., Lactobacillus rhamnosus GG-supplemented formula expands butyrate-producing bacterial strains in food allergic infants. 2015; Vol. 10.

560 47. Pimentel, G.; Burton, K. J.; Rosikiewicz, M.; Freiburghaus, C.; Von, A. U.; Münger, L. H.; 561 Pralong, F. P.; Vionnet, N.; Greub, G.; Badertscher, R., Blood lactose after dairy product intake 562 in healthy men. British Journal of Nutrition 2017, 118 (12), 1070.

563 48. Carles, U.; Vanni, B.; Silvia, C.; Ana, D.; Toussaint, N. C.; Michele, E.; Lauren, L.; Lilan, 564 L.; Asia, G.; Daniel, N., Intestinal microbiota containing Barnesiella species cures vancomycin565 resistant Enterococcus faecium colonization. Infection \& Immunity 2013, 81 (3), 965-973.

566 49. Anhê, F. F.; Nachbar, R. T.; Varin, T. V.; Vilela, V.; Dudonné, S.; Pilon, G.; Fournier, M.; 567 Lecours, M. A.; Desjardins, Y.; Roy, D., A polyphenol-rich cranberry extract reverses insulin 568 resistance and hepatic steatosis independently of body weight loss. Molecular Metabolism

569

570

571

572

573

574

575

576

577

578

579

580

581

582

583

584

585

586

587

588

589

590

591

592

593

594

595

596

597 2017, 6 (12), 1563-1573.

50. $\quad$ Cani, P. D.; Rodrigo, B.; Claude, K.; Aurélie, W.; Neyrinck, A. M.; Delzenne, N. M.; Rémy, B., Changes in gut microbiota control metabolic endotoxemia-induced inflammation in high-fat diet-induced obesity and diabetes in mice. Diabetes 2008.

51. Bäckhed, F.; Normark, S.; Schweda, E. K. H.; Oscarson, S.; Richter-Dahlfors, A., Structural requirements for TLR4-mediated LPS signalling: a biological role for LPS modifications. Microbes \& Infection 2003, 5 (12), 1057-1063.

52. Cani, P.; Amar, J.; Iglesias, M.; Poggi, M.; Knauf, C.; Bastelica, D.; Neyrinck, A.; Fava, F.; Tuohy, K.; Chabo, C.; Waget, A.; Delmée, E.; Cousin, B.; Sulpice, T.; Chamontin, B.; Ferrières, J.; Tanti, J.-F.; R Gibson, G.; Casteilla, L.; Burcelin, R., Metabolic Endotoxemia Initiates Obesity and Insulin Resistance. 2007; Vol. 56, p 1761-72.

53. Cao, J.; Peng, J.; An, H.; He, Q.; Boronina, T.; Guo, S.; White, M. F.; Cole, P. A.; He, L., Endotoxemia-mediated activation of acetyltransferase P300 impairs insulin signaling in obesity. Nature Communications 2017, 8 (1), 131.

54. Pussinen, P. J.; Havulinna, A. S.; Lehto, M.; Sundvall, J.; Salomaa, V., Endotoxemia is associated with an increased risk of incident diabetes. Diabetes care 2011, 34 (2), 392-397.

55. Adams, C. A., The probiotic paradox: live and dead cells are biological response modifiers. Nutrition research reviews 2010, 23 (1), 37-46.

56. Clarke, C.; Hales, A.; Hunt, A.; Foxwell, B., IL-10-mediated suppression of TNF-alpha production is independent of its ability to inhibit NF kappa B activity. European Journal of Immunology 2015, 28 (5), 1719-1726.

57. Fiorentino, D. F.; Zlotnik, A.; Mosmann, T. R.; Howard, M.; O'Garra, A., IL-10 inhibits cytokine production by activated macrophages. Journal of Immunology 1991, 147 (11), 3815-22. 58. Tazuke, Y.; Wasa, M.; Satoko, N.; Fukuzawa, M., Protective mechanism of glutamine on the expression of proliferating cell nuclear antigen after cisplatin-induced intestinal mucosal injury. Pediatric Surgery International 2011, 27 (2), 151-158.

59. Abimosleh, S. M.; Lindsay, R. J.; Butler, R. N.; Cummins, A. G.; Howarth, G. S., Emu oil increases colonic crypt depth in a rat model of ulcerative colitis. Digestive Diseases \& Sciences 2012, 57 (4), 887-896.

Peer] reviewing PDF | (2019:01:34665:2:2:NEW 3 May 2019) 
598 60. Catherine, R.; Calliope, C.; Bernadette, C.; Jean-Claude, M., Structural diversity and

599

600

601

602

603

604

605

606

607

608

609

610

611

612

613

614

615

616

617

618

619

620

621

622

623

624

625

626

627

628

629

630

631

632

633

634

635

636

637

638

639

640

641

642

643

644

645

646

647 specific distribution of O-glycans in normal human mucins along the intestinal tract. Biochemical Journal 2004, 384 (2), 307-16.

61. Yeung, C. Y.; Chan, W. T.; Jiang, C. B.; Cheng, M. L.; Liu, C. Y.; Chang, S. W.; Chiau, J. S. C.; Lee, H. C., Amelioration of Chemotherapy-Induced Intestinal Mucositis by Orally Administered Probiotics in a Mouse Model. PloS one 2015, 10 (9), e0138746.

62. Leiper, K.; Campbell, B. J.; Jenkinson, M. D.; Milton, J.; Lugang, Y. U.; Democratis, J.; Rhodes, J. M., Interaction between bacterial peptides, neutrophils and goblet cells: a possible mechanism for neutrophil recruitment and goblet cell depletion in colitis. Clinical Science 2001, 101 (4), 395-402.

63. Zweimueller-Mayer, J.; Steinbacher, P.; Lametschwandtner, A.; Bauer, H.-C., The Dual Role of Zonula Occludens (ZO) Proteins. 2010; Vol. 2010, p 402593.

64. Alam, M. A.; Neish, A., Role of gut microbiota in intestinal wound healing and barrier function. 2018; Vol. 6, p 1-22.

65. Cani, P.; Possemiers, S.; Van de Wiele, T.; Guiot, Y.; Everard, A.; Rottier, O.; Geurts, L.; Naslain, D.; Neyrinck, A.; Lambert, D.; Muccioli, G.; Delzenne, N., Changes in Gut Microbiota Control Inflammation in Obese Mice through a Mechanism Involving GLP-2-Driven Improvement of Gut Permeability. 2009; Vol. 58, p 1091-103.

66. Terpend, K.; Blaton, M. A.; Candalh, C.; Wal, J. M.; Pochart, P.; Heyman, M., Intestinal barrier function and cow's milk sensitization in guinea pigs fed milk or fermented milk. Journal of Pediatric Gastroenterology \& Nutrition 1999, 28 (2), 191-8.

67. Resta-Lenert, S.; Barrett, K. E., Live probiotics protect intestinal epithelial cells from the effects of infection with enteroinvasive Escherichia coli (EIEC). Gut 2003, 52 (7), 988-997.

68. Plovier, H.; Everard, A.; Druart, C.; Depommier, C.; Van Hul, M.; Geurts, L.; Chilloux, J.; Ottman, N.; Duparc, T.; Lichtenstein, L.; Myridakis, A.; Delzenne, N.; Klievink, J.; Bhattacharjee, A.; van der Ark, K.; Aalvink, S.; Martinez, L.; Dumas, M.-E.; Maiter, D.; Cani, P., A purified membrane protein from Akkermansia muciniphila or the pasteurized bacterium improves metabolism in obese and diabetic mice. 2016; Vol. 23.

69. Zorzela, L.; Ardestani, S. K.; McFarland, L. V.; Vohra, S., Is there a role for modified probiotics as beneficial microbes: a systematic review of the literature. Beneficial microbes 2017, 8 (5), 1-16.

70. Ménard, S.; Candalh, C.; Bambou, J. C.; Terpend, K.; Cerf-Bensussan, N.; Heyman, M., Lactic acid bacteria secrete metabolites retaining anti-inflammatory properties after intestinal transport. Gut 2004, 53 (6), 821.

71. Chelakkot, C.; Choi, Y.; Kim, D. K.; Park, H. T.; Ghim, J.; Kwon, Y.; Jeon, J.; Kim, M. S.; Jee, Y. K.; Gho, Y. S., Akkermansia muciniphila-derived extracellular vesicles influence gut permeability through the regulation of tight junctions. Experimental \& Molecular Medicine 2018, 50 (2), e450.

72. Cavallari, J. F.; Fullerton, M. D.; Duggan, B. M.; Foley, K. P.; Denou, E.; Smith, B. K.; Desjardins, E. M.; Henriksbo, B. D.; Kim, K. J.; Tuinema, B. R., Muramyl Dipeptide-Based Postbiotics Mitigate Obesity-Induced Insulin Resistance via IRF4. Cell metabolism 2017, 25 (5), 1063-1074.

73. Beaumont, M.; Neyrinck, A. M.; Olivares, M.; Rodriguez, J.; Nathalie, M. D., The gut microbiota metabolite indole alleviates liver inflammation in mice. Faseb Journal 2018, 32 (12), fj.201800544.

74. Elamin, E. E.; Masclee, A. A.; Jan, D.; Harm-Jan, P.; Jonkers, D. M., Short-Chain Fatty Acids Activate AMP-Activated Protein Kinase and Ameliorate Ethanol-Induced Intestinal Barrier Dysfunction in Caco-2 Cell Monolayers. Journal of Nutrition 2013, 143 (12), 1872-1881.

75. L. Sonnenburg, J.; Bäckhed, F., Diet-microbiota interactions as moderators of human metabolism. 2016; Vol. 535, p 56-64.

Peer] reviewing PDF | (2019:01:34665:2:2:NEW 3 May 2019) 
648 76. Canfora, E.; W Jocken, J.; Blaak, E., Short-chain fatty acids in control of body weight 649 and insulin sensitivity. 2015; Vol. 11.

650 77. De, V. F.; Kovatchevadatchary, P.; Zitoun, C.; Duchampt, A.; Bäckhed, F.; Mithieux, G., 651 Microbiota-Produced Succinate Improves Glucose Homeostasis via Intestinal Gluconeogenesis. 652 Cell metabolism 2016, 24 (1), 151-157.

653 78. Mhrouster-Stevens, L., Lactobacillus sepsis associated with probiotic therapy. Journal of 654 Urology 2005, 174 (5), 1843-1843.

655 79. Berger, R. E., Lactobacillus Sepsis Associated With Probiotic Therapy. Journal of 656 Urology 2005, $174(5), 1843-1843$.

657 80. Vintiñi, E. O.; Medina, M. S., Host immunity in the protective response to nasal 658 immunization with a pneumococcal antigen associated to live and heat-killed Lactobacillus 659 casei. BMC Immunology, 12, 1(2011-08-11) 2011, 12 (1), 46-46.

660 81. Lick, S.; Drescher, K.; Heller, K. J., Survival of Lactobacillus delbrueckii subsp. 661 bulgaricus and Streptococcus thermophilus in the terminal ileum of fistulated G?ttingen 662 minipigs. Applied \& Environmental Microbiology 2001, 67 (9), 4137-43.

663 82. Josef, N.; Ricardo, C., Probiotics: protecting the intestinal ecosystem? Journal of 664 Pediatrics 2005, 147 (2), 143-146.

665 83. Gao, Z.; Li, Q.; Wu, X.; Zhao, X.; Zhao, L.; Tong, X., New Insights into the Mechanisms 666 of Chinese Herbal Products on Diabetes: A Focus on the "Bacteria-Mucosal Immunity667 Inflammation-Diabetes” Axis. Journal of Immunology Research 2017, 2017, 1-13. 


\section{Table $\mathbf{1}$ (on next page)}

The differences in some variables between the DM+ST and DM groups

$\mathrm{N}=5$ in each group. Data represented as means $\pm \mathrm{SD}$. ALT: Alanine aminotransferase, TC:

Total cholesterol, HDL-C: High-density lipoprotein cholesterol, LDL-C: Low-density lipoprotein cholesterol, LNIL6: Ln transformation of interleukin-6, LNIL10: Ln transformation of interleukin-10, LnTNF- $\alpha$ : Ln transformation oftumornecrosisfactor- $\alpha$, LPS: L ipopolysaccharide , HOMA-IR: Homeostasis model assessment of insulin resistance, FBG: Fasting blood glucose. The heat-killed $S$. thermophilus treatment reduced TC, LPS, IL-6, IL-10, TNF- $\alpha$, fasting insulin levels, HbAlc, FBG, and HOMA-IR in ZDF diabetic rats; *P < 0:05. 


\begin{tabular}{|c|c|c|c|c|}
\hline Variables & DM & $\mathrm{DM}+\mathrm{ST}$ & $\mathrm{t}$ & $\mathrm{p}$ \\
\hline Weight(g) & $354.2 \pm 35.2$ & $360.2 \pm 33.0$ & -0.3 & 0.788 \\
\hline Creatinine (umol/l $)$ & $16.0 \pm 6.2$ & $15.0 \pm 2.1$ & 0.3 & 0.742 \\
\hline $\mathrm{ALT}(\mathrm{U} / \mathrm{L})$ & $119.4 \pm 51.5$ & $123.4 \pm 52.7$ & -0.1 & 0.906 \\
\hline Carbamide $(\mathrm{mmol} / \mathrm{l})$ & $6.0 \pm 0.8$ & $7.2 \pm 1.6$ & -1.6 & 0.159 \\
\hline Uric acid(umol/l) & $116.2 \pm 32.6$ & $122.6 \pm 18.2$ & -0.4 & 0.711 \\
\hline $\mathrm{TC}(\mathrm{mmol} / \mathrm{l})$ & $5.5 \pm 0.4$ & $4.7 \pm 0.2$ & 4.1 & $0.003 *$ \\
\hline Triglyceride (mmol/l) & $3.0 \pm 0.9$ & $2.5 \pm 0.3$ & 1.2 & 0.304 \\
\hline HDL-C(mmol/l) & $2.7 \pm 0.2$ & $2.5 \pm 0.2$ & 1.8 & 0.115 \\
\hline LDL-C(mmol/l) & $1.2 \pm 0.2$ & $0.9 \pm 0.1$ & 2.5 & 0.063 \\
\hline LPS(ng/ml) & $0.7 \pm 0.1$ & $0.5 \pm 0.1$ & 2.9 & $0.019 *$ \\
\hline LNIL6(pg/ml) & $4.6 \pm 0.6$ & $3.9 \pm 0.3$ & 2.7 & $0.038 *$ \\
\hline LNIL10(pg/ml) & $4.0 \pm 0.3$ & $4.5 \pm 0.3$ & -2.4 & $0.046^{*}$ \\
\hline LnTNF-a(pg/ml) & $4.1 \pm 0.3$ & $3.6 \pm 0.2$ & 3.0 & $0.017 *$ \\
\hline Fasting insulin (ulU/ml) & $107.6 \pm 18.1$ & $67.0 \pm 8.3$ & 4.6 & $0.002 *$ \\
\hline HOMA-IR & $106.7 \pm 25.5$ & $40.8 \pm 3.8$ & 5.7 & $0.004 *$ \\
\hline $\mathrm{HbA} 1 \mathrm{c}(\%)$ & $12.0 \pm 2.0$ & $8.6 \pm 1.2$ & 3.3 & $0.011^{*}$ \\
\hline $\mathrm{FBG}(\mathrm{mmol} / \mathrm{L})$ & $22.3 \pm 3.6$ & $13.7 \pm 1.0$ & 3.1 & $0.036^{*}$ \\
\hline
\end{tabular}

1 


\section{Table 2 (on next page)}

The differences in some variables between the CON+ST and CON groups

$\mathrm{N}=5$ in each group. Data represented as means $\pm \mathrm{SD}$. ALT: Alanine aminotransferase, TC:

Total cholesterol, HDL-C: High-density lipoproteincholesterol, LDL-C: Low-density lipoproteincholesterol, LNIL6: Ln transformation of interleukin-6, LNIL10: Ln transformation of interleukin-10, LnTNF- $\alpha$ : Ln transformation of tumornecrosisfactor- $\alpha$, LPS: Llipopolysaccharide , HOMA-IR: Homeostasis model assessment of insulin resistance, FBG: Fasting blood glucose. Compared to the CON group, the heat-killed S. thermophilus treatment rats failed to show significant variations. 


\begin{tabular}{lcccc}
\hline \multicolumn{1}{c}{ Variables } & CON+ST & CON & $t$ & $\mathrm{p}$ \\
\hline Weight(g) & $503.2 \pm 48.5$ & $476.8 \pm 37.3$ & 1.0 & 0.363 \\
Creatinine(umol/l) & $24.2 \pm 2.8$ & $26.2 \pm 4.7$ & -0.8 & 0.437 \\
ALT(U/L) & $37.6 \pm 9.8$ & $36.8 \pm 7.6$ & 0.1 & 0.889 \\
Carbamide(mmol/l) & $6.1 \pm 1.4$ & $5.7 \pm 1.5$ & 0.4 & 0.709 \\
Uric acid(umol/l) & $122.8 \pm 21.7$ & $154.0 \pm 66.6$ & -1.0 & 0.348 \\
TC(mmol/l) & $1.4 \pm 0.3$ & $1.8 \pm 0.4$ & -1.8 & 0.11 \\
Triglyceride (mmol/l) & $0.7 \pm 0.2$ & $0.7 \pm 0.3$ & 0.1 & 0.963 \\
HDL-C(mmol/l) & $0.8 \pm 0.2$ & $0.9 \pm 0.2$ & -1.3 & 0.246 \\
LDL-C(mmol/l) & $0.3 \pm 0.1$ & $0.4 \pm 0.1$ & -1.6 & 0.16 \\
LPS(ng/ml) & $0.4 \pm 0.1$ & $0.4 \pm 0.1$ & -1.0 & 0.369 \\
InIn6(pg/ml) & $4.0 \pm 0.2$ & $4.1 \pm 0.3$ & -0.6 & 0.585 \\
InlL10(pg/ml) & $4.3 \pm 0.5$ & $4.1 \pm 0.1$ & 0.8 & 0.465 \\
InTNF- $\alpha(\mathrm{pg} / \mathrm{ml})$ & $3.9 \pm 0.4$ & $4.1 \pm 0.3$ & -1.1 & 0.323 \\
Fasting insulin (ulU/ml) & $41.1 \pm 7.0$ & $42.2 \pm 12.5$ & -0.2 & 0.863 \\
FBG $(\mathrm{mmol} / \mathrm{L})$ & $6.7 \pm 0.6$ & $6.9 \pm 0.6$ & -0.4 & 0.72 \\
HOMA-IR & $12.3 \pm 2.8$ & $12.7 \pm 2.9$ & -0.2 & 0.857 \\
\hline
\end{tabular}

1 


\section{Table 3 (on next page)}

Alpha diversity indices

Data are presented as means \pm SD $(n=5)$. In ZDF diabetic rats, the richness of the gut microbiota was increased in the DM+ST group compared with the DM group; however, the difference was not significant ( $P>0.05, t$ test and Wilcoxon rank-sum test). Significant difference did not exist between the CON+ST group and the CON group ( $P>0.05, t$ test and Wilcoxon rank-sum test) . 


\begin{tabular}{lcccc}
\hline & CON & CON + ST & DM & DM+ST \\
\hline OTUs & $1497.40 \pm 327.41$ & $1504.20 \pm 275.38$ & $1064.60 \pm 230.90$ & $1298.60 \pm 323.53$ \\
Chao1 & $4143.66 \pm 490.57$ & $4218.70 \pm 524.60$ & $3253.11 \pm 518.16$ & $3185.49 \pm 733.41$ \\
Shannon & $117.34 \pm 11.09$ & $121.01 \pm 11.65$ & $86.96 \pm 8.13$ & $90.94 \pm 20.40$ \\
Simpson & $6.76 \pm 0.66$ & $6.88 \pm 0.39$ & $4.92 \pm 0.70$ & $5.72 \pm 1.22$ \\
PD_whole_tree & $0.93 \pm 0.04$ & $0.93 \pm 0.02$ & $0.81 \pm 0.08$ & $0.87 \pm 0.09$ \\
\hline
\end{tabular}

1 
Figure 1 (on next page)

The effect of heat-killed S.thermophilus on blood glucose during the OGTT in the DM+ST and DM groups

OGTT: oral glucose tolerance test. Error bars represent one standard deviation. The blood glucose levels significantly decreased before and 15, 60, and $90 \mathrm{~min}$ after glucose load in the $\mathrm{DM}+\mathrm{ST}$ group as compared to those in the DM group according to the OGTT. * $\mathrm{p}<0.05$. 


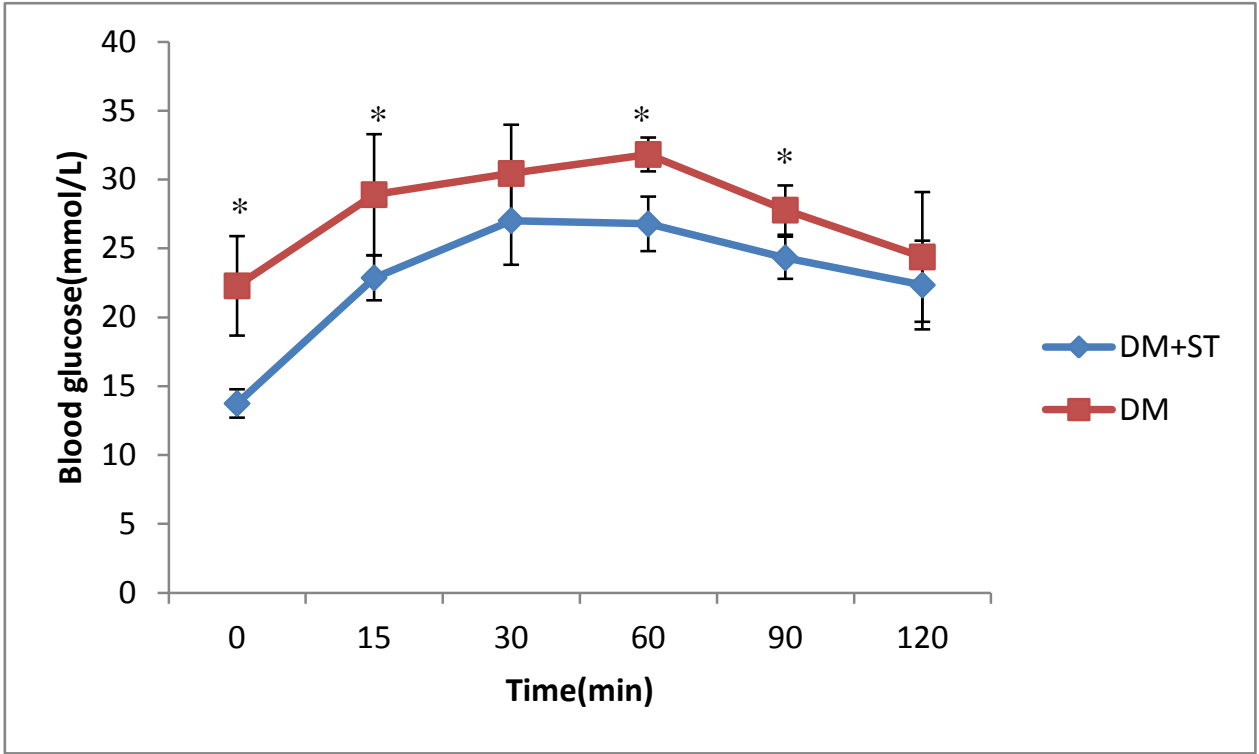


Figure 2 (on next page)

Area under the curve (AUC) for the OGTT in the DM+ST and DM groups

Compared with the DM group, the glucose area under the curve (AUC) for the OGTT in the DM+ST group exhibited a reduced glucose AUC by $14.7 \% ; * P<0.05$. 


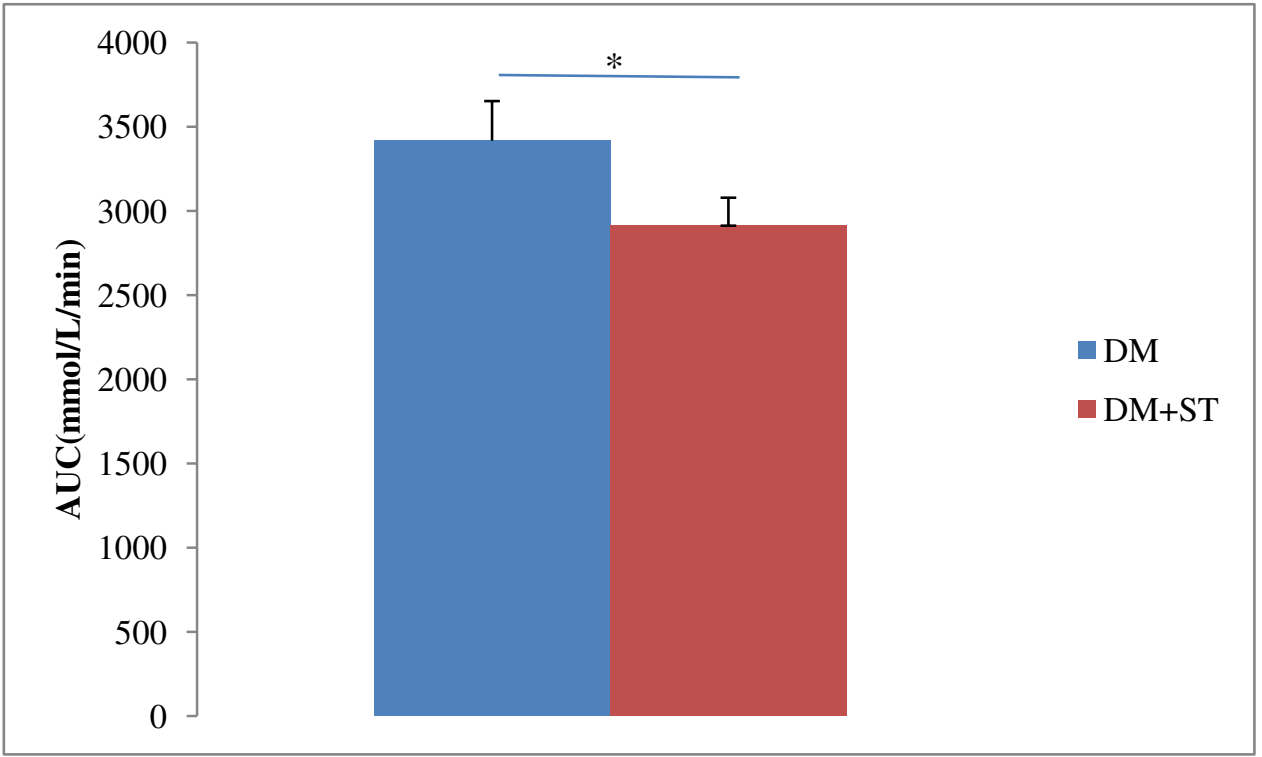


Figure 3 (on next page)

The effect of heat-killed S.thermophilus on blood glucose during the OGTT in the $\mathrm{CON}+\mathrm{ST}$ and CON groups

OGTT: oral glucose tolerance test. Error bars represent one standard deviation. According to the OGTT, although there were no significant differences before and 15, and 120 min after the glucose load between the CON group and CON+ST group, the blood glucose levels significantly decreased 30 and $60 \mathrm{~min}$ after the glucose load in the CON+ST group as compared to those in the CON group. $* p<0.05$. 


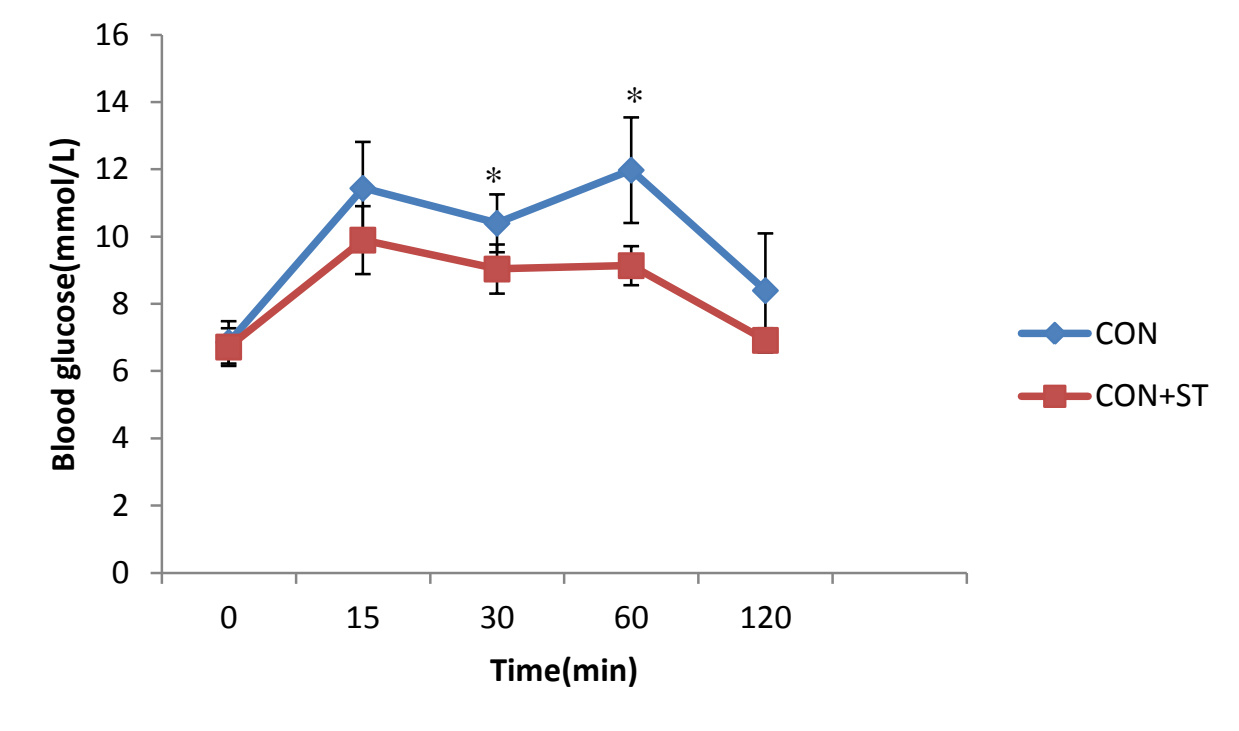


Figure 4(on next page)

Area under the curve (AUC) for the OGT in the CON+ST and CON groups

The CON+ST group exhibited a reduced glucose AUC by $18.2 \%$ for the OGTT, compared with the CON group; ${ }^{*} P<0.05$. 


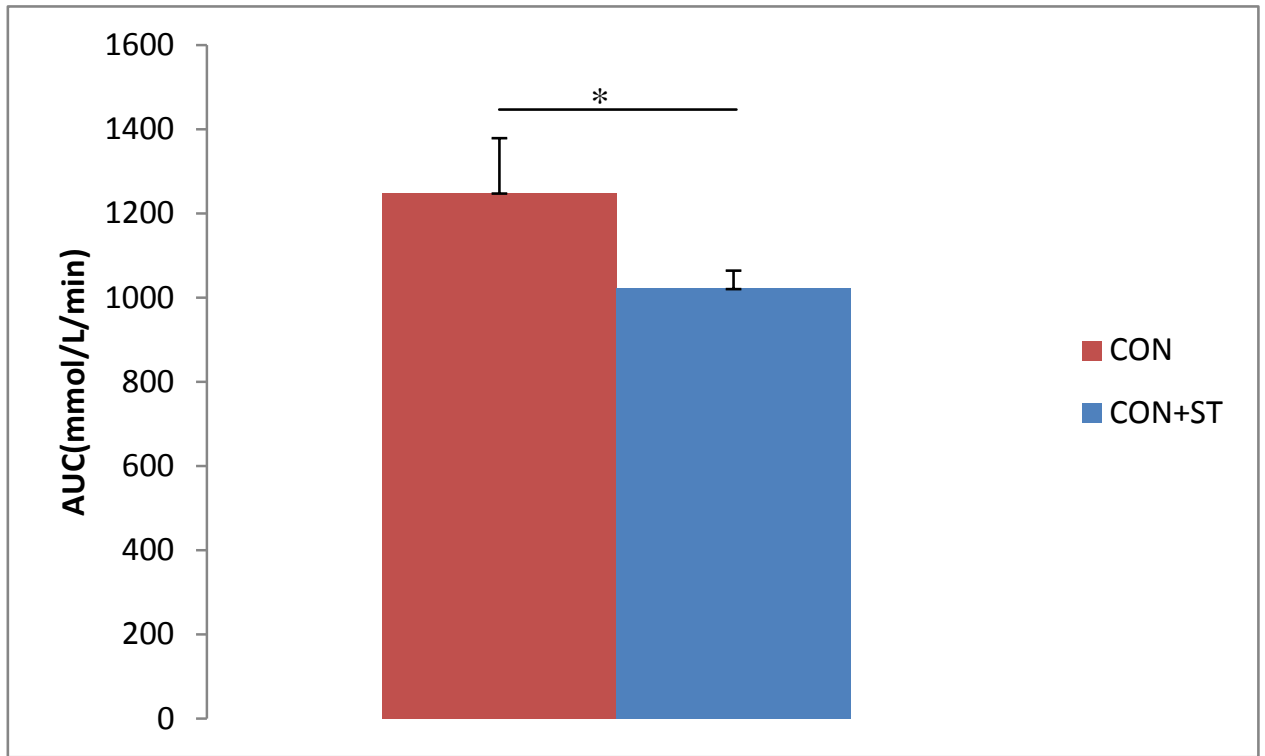




\section{Figure 5}

Representative histology of the ileum and colon with HE stain in T2D model rats.

(A) Histology of the ileum in the DM group, (B) histology of the ileum in the DM+ST group, (C) histology of the colon in the DM group, and (D) histology of the colon in the DM+ST group. The image acquisition phase was performed with a 50X objective. Scale bar $=200 \mu \mathrm{m}$. In the diabetic rats, the intestinal mucosal layer was characterized by disturbed mucosal architecture, shortened villi, blunted villus tips, and inflammatory cell infiltration. In the $\mathrm{DM}+\mathrm{ST}$ group, oral administration of $\mathrm{S}$. thermophilus restored the normal structure of the intestinal mucosal layer. 


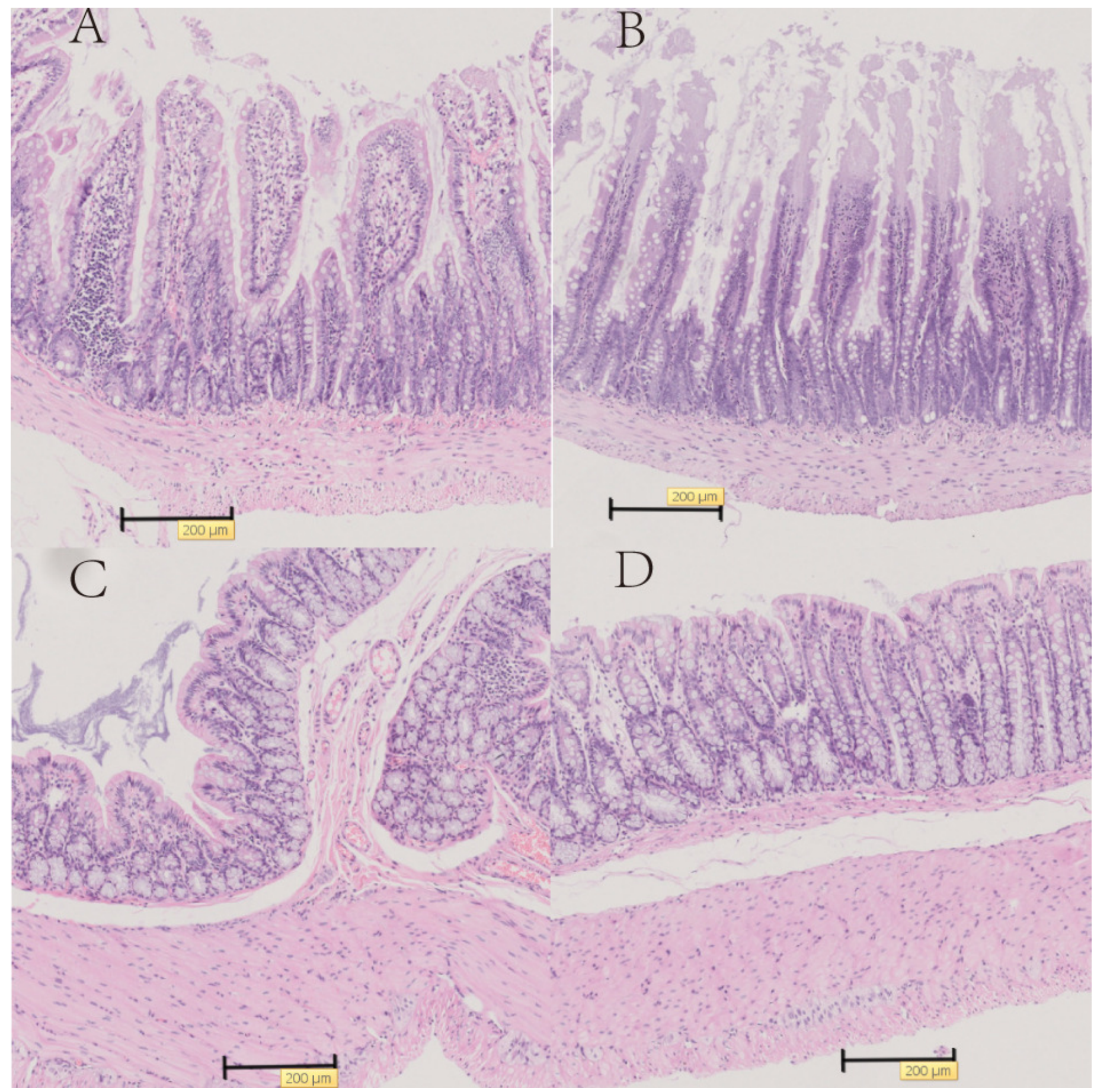


Figure 6 (on next page)

The length of villi and depth of crypts in the ileum of diabetic rats

The length of villi and depth of crypts in the ileum in the DM+ST group were significantly increased compared to those in DM group; $* P<0.05$. 


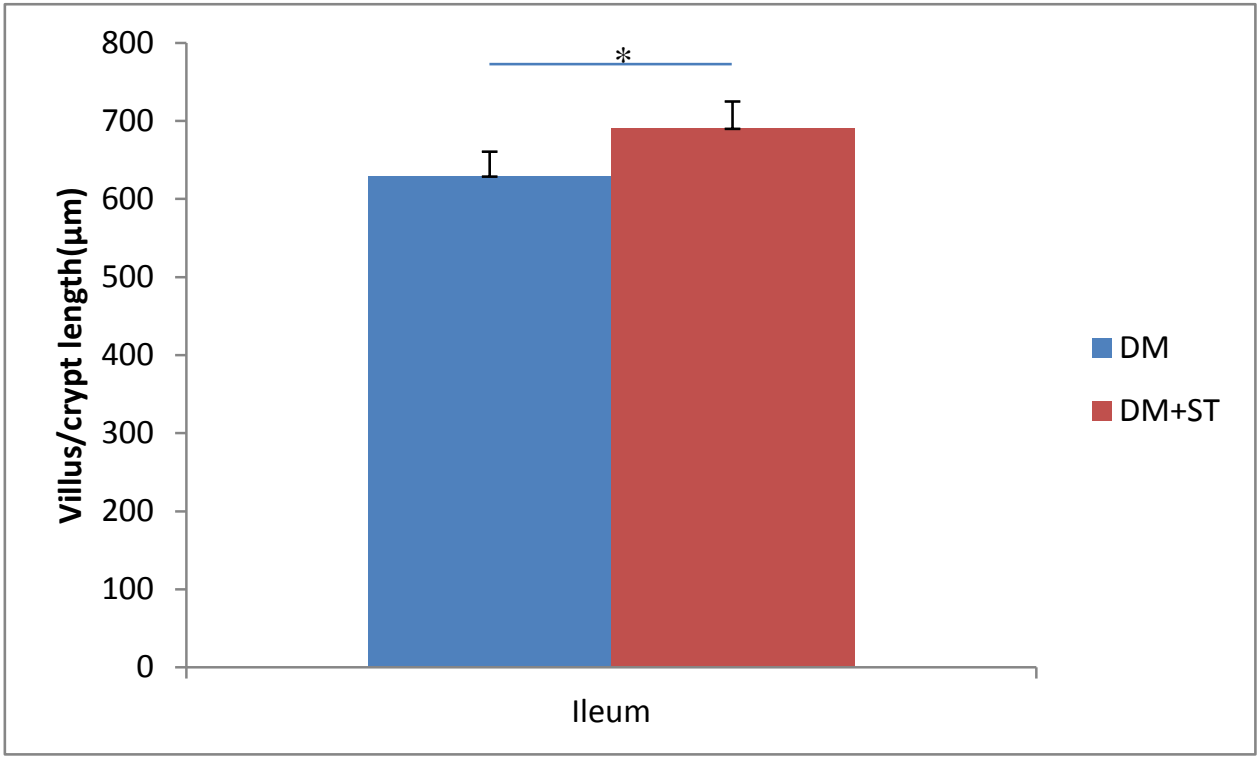


Figure 7 (on next page)

The length of villi and depth of crypts in the colon of diabetic rats

The length of villi and depth of crypts in the colon in the DM+ST group were significantly increased compared to those in DM group; $* P<0.05$. 


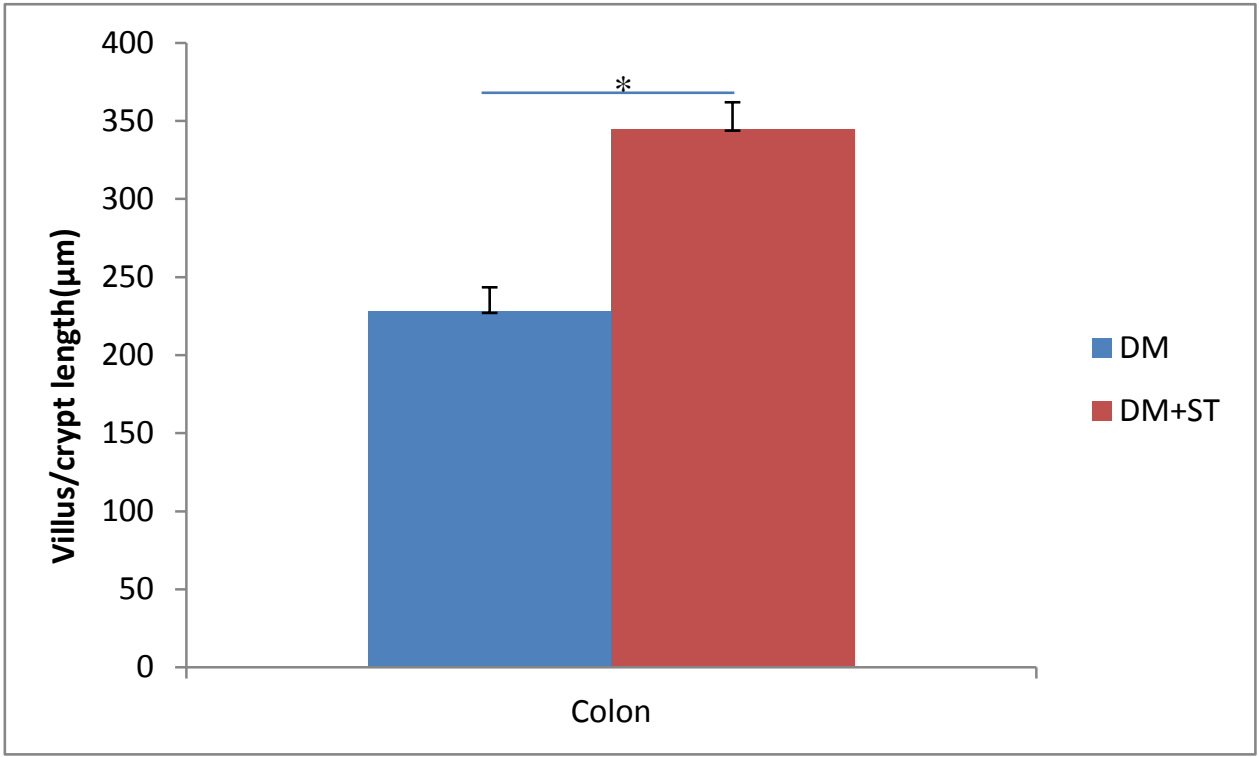




\section{Figure 8}

Representative histology of the ileum and colon with HE stain in SD rats

(A) Histology of ileum in the CON group, (B) Histology of ileum in the CON+ST group, (C)

Histology of colon in the CON group, (D) Histology of colon in the CON+ST group. The image acquisition phase was performed with a 50X objective. Scale bar $=200 \mu \mathrm{m}$. The characteristics of the intestinal mucosal layer were similar between the CON+ST group and CON group. There were no differences in the villi length and crypt depth and the numbers of goblet cells between the CON+ST group and CON group. 


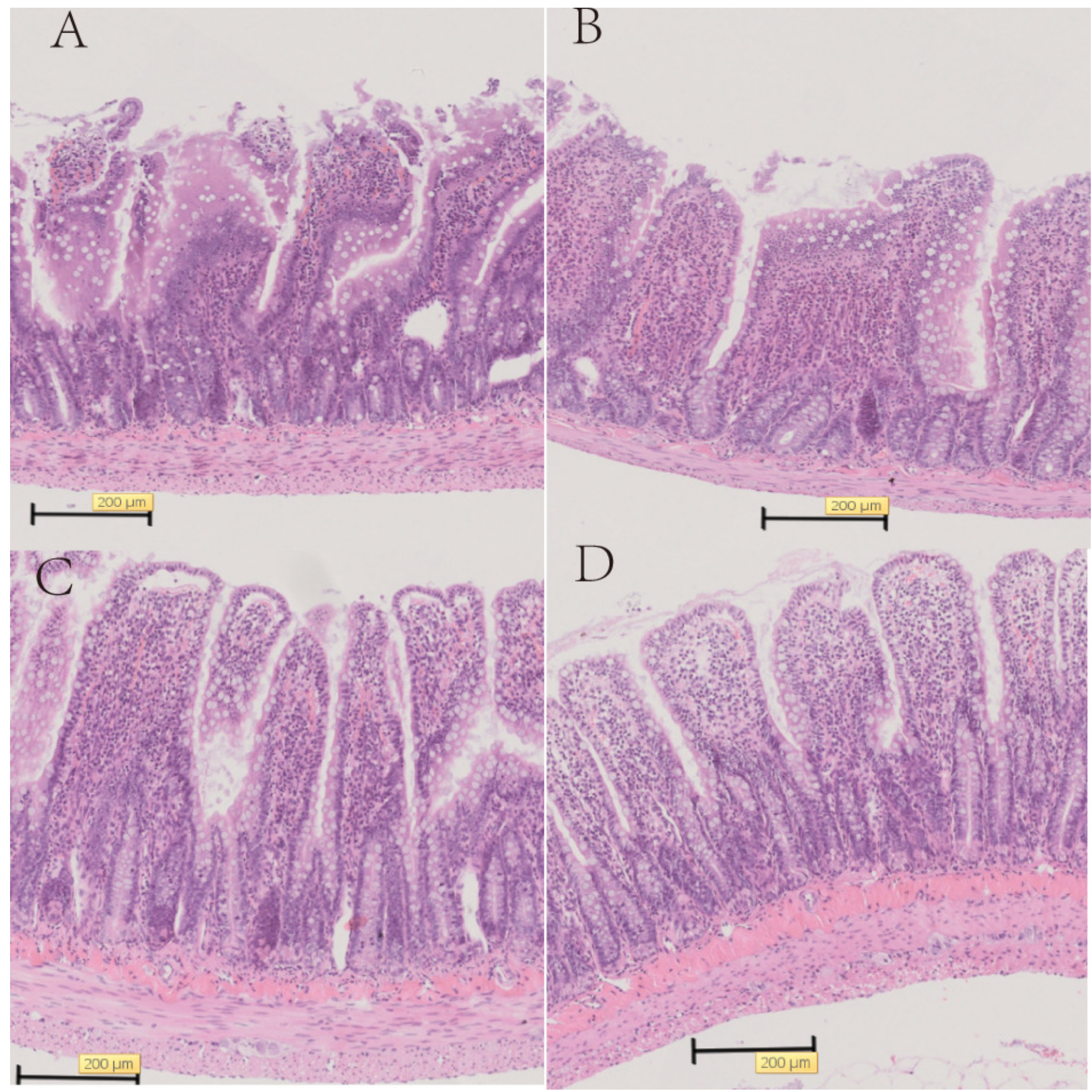


Figure 9 (on next page)

Effects of the heat-killed $S$. thermophilus treatment on tight junction proteins in the $\mathrm{DM}+\mathrm{ST}$ and DM groups.

(A) lleum and colon extracts from DM+ST and DM groups were used for Western blot analysis; (B) Expression levels of Occludin were quantified by measuring band densities; (C) Expression levels of ZO-1 were quantified by measuring band densities. $\beta$-actin was used as a loading control. $* p<0.05$. 


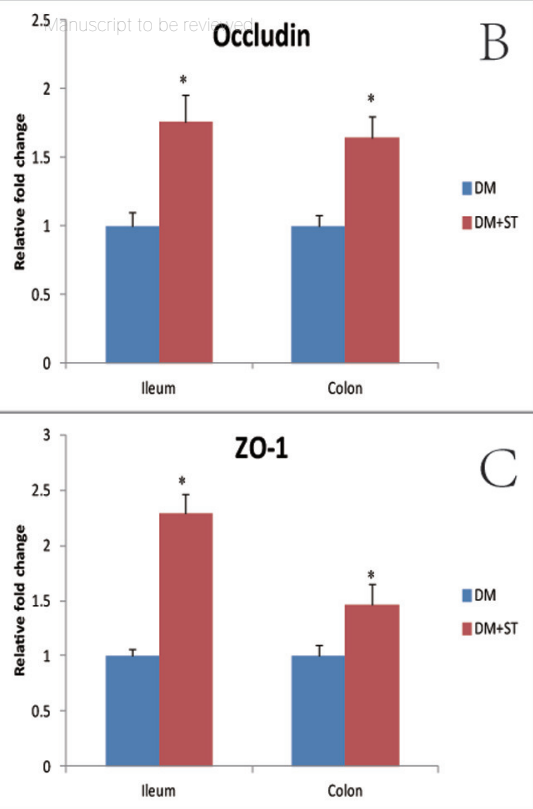


Figure 10 (on next page)

Effects of the heat-killed $S$. thermophilus treatment on tight junction proteins in the $\mathrm{CON}+\mathrm{ST}$ and CON groups

(A) lleum and colon extracts from CON+ST and CON groups were used for Western blot analysis; (B) Expression levels of Occludin were quantified by measuring band densities; (C) Expression levels of ZO-1 were quantified by measuring band densities. $\beta$-actin was used as a loading control. 


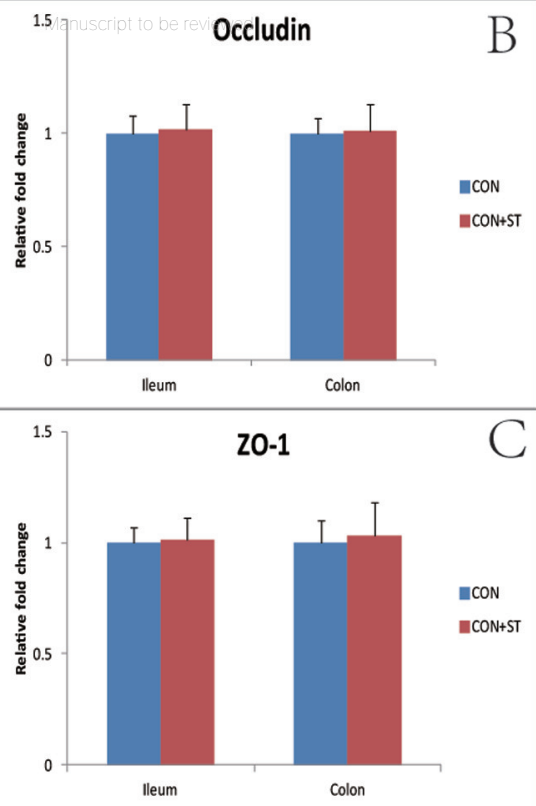


Figure 11

PCoA of unweighted UniFrac distances of the gut bacterial communities between the $\mathrm{DM}+\mathrm{ST}$ and DM groups

The first two principal coordinates of PCoA were separated into DM+ST and DM groups, which shared overlapping regions.

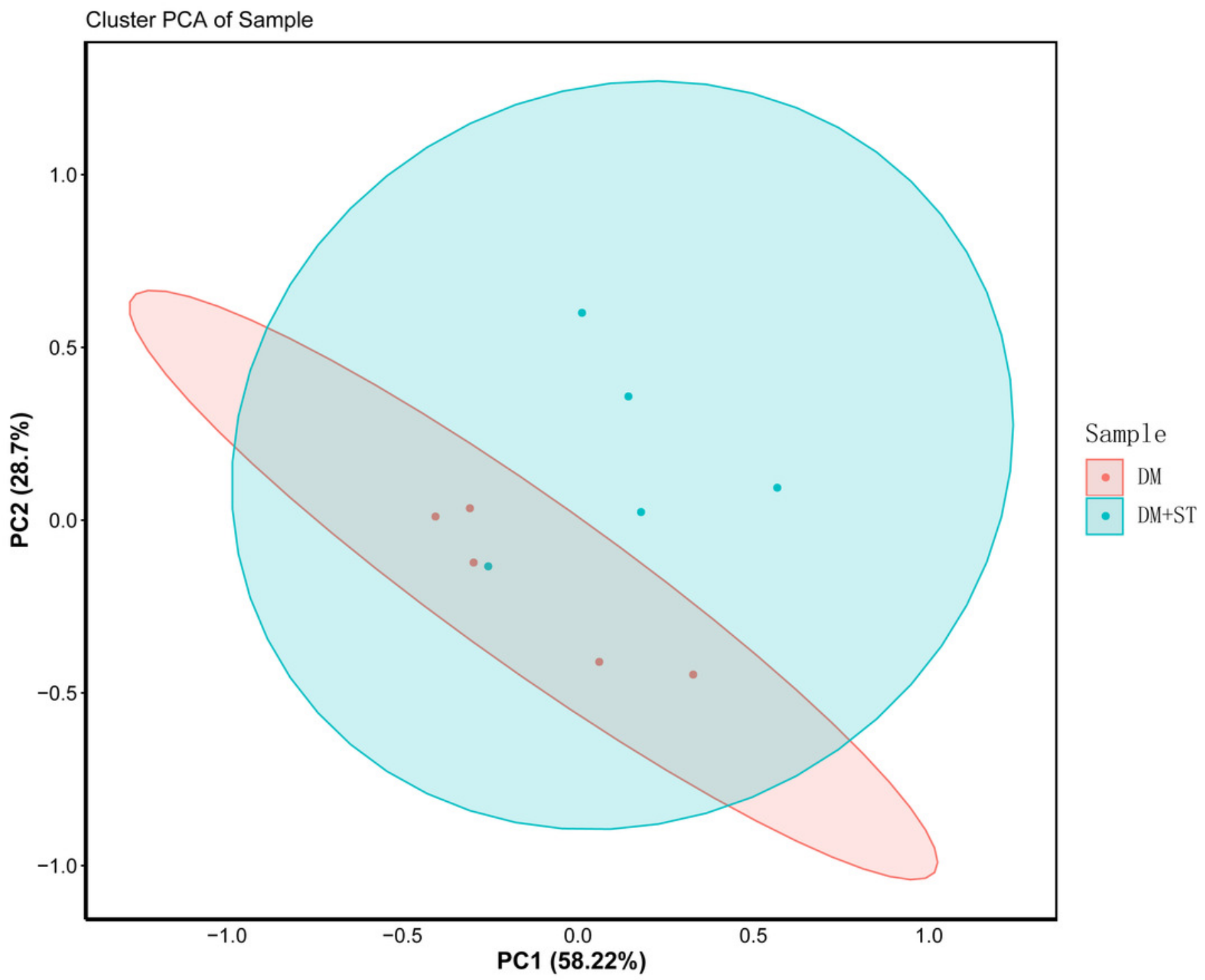


Figure 12 (on next page)

The $t$ test results of the relative abundance (\%) of bacteria from the DM+ST and DM groups.

At the genus level, the abundance of Ruminococcaceae, Veillonella, Coprococcus, and Bamesiella was significantly elevated by heat-killed S. thermophilus treatment in ZDF diabetic rats. 
$95 \%$ confidence intervals

Otherf_Ruminococcaceae

g_Veillonella

g_Coprococcus

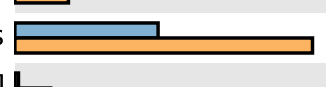

g_f_[Barnesiellaceae]

$$
0.2
$$

HO- $1.20 \mathrm{e}-3$ $\longmapsto 0.015$ 0.032 $\longmapsto 0.046$ 0.0

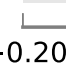

$-0.15$ Difference in mean proportions (\%) 
Figure 13(on next page)

The $t$ test results of the relative abundance (\%) of bacteria from the CON+ST and CON groups

Compared with the CON group, Phascolarctobacterium and Dorea abundances were reduced by heat-killed $S$. thermophilus treatment in the CON+ST group. 
$95 \%$ confidence intervals

g_Phascolarctobacterium

g_Enterococcus |

g_f_Clostridiaceae p

g_Dorea $P$

g_c_Clostridia

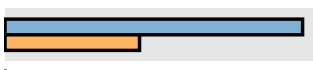

ard

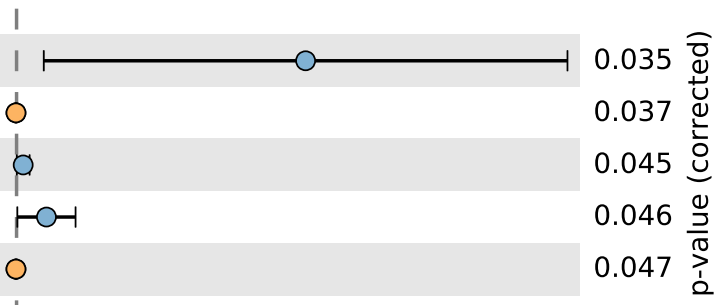

0.0

$2.3-0.5$

0.0

0.5

1.0

1.5

2.0

2.5

Mean proportion (\%)

Difference in mean proportions (\%) 